\title{
UNIFORM ASYMPTOTIC SOLUTIONS FOR LAMELLAR INHOMOGENEITIES IN PIEZOELECTRIC SOLIDS
}

\author{
BY \\ CRISTIAN DASCALU (Laboratoire Sols, Solides, Structures, UMR5521 CNRS, UJF-INPG, BP. \\ 53, 38041 Grenoble, France) \\ AND
}

DOREL HOMENTCOVSCHI (Institute of Applied Mathematics of the Romanian Academy, P.O. Box 1-24, RO-70700, Bucharest, Romania)

\begin{abstract}
We study the problem of a lamellar inhomogeneity of arbitrary shape embedded in a piezoelectric matrix of infinite extent. Uniform asymptotic solutions for the equations of elastostatics and electrostatics on this configuration are obtained. The first order terms, in the inhomogeneity thickness, are explicitly determined for piezoelectric inclusions, rigid inclusions of electric conductor, impermeable cracks, and cracks with inside electric field. We give real-form expressions of mechanical and electric fields at the interface and on the inhomogeneity axis. Detailed first order solutions are obtained for elliptic and lemon-shaped inhomogeneities. It is found that, while for elliptic piezoelectric inclusions the perturbation stresses and electric displacements at the inclusion ends have the same order as those given at infinity, for a lemon-shaped inclusion they are an order-of-magnitude smaller. Intensity factors are calculated for lemon-shaped cavities. It is shown that, when inside electric fields are considered, the stress intensity coefficients are influenced by the material anisotropy.
\end{abstract}

1. Introduction. In recent years, a lot of effort has been expended for the study of inhomogeneities, like inclusions or cracks, in piezoelectric materials (e.g., Pak 1990, Sosa 1991, Suo et al. 1992. Liang et al. 1995, Park and Sun 1995, Dascalu and Maugin 1995, Sosa and Khutoryansky 1996, Chung and Ting 1996, Dascalu 1997, Zhang et al. 1998, Lu et al. 2000). However, most of the obtained results concern the cases of elliptic inclusions or line cracks. The objective of this paper is to give solutions for arbitraryshaped lamellar inhomogeneities. We consider inclusions embedded in an infinite matrix subject to a uniform stress at infinity. Both the matrix and the inclusion consist of general piezoelectric materials. We also consider the case of a rigid inclusion of electric conductor and that of a cavity embedded in a piezoelectric material.

Received January 30, 2001.

2000 Mathematics Subject Classification. Primary 74B05, 74F15, 74E10, 74G10, 74G70.

(C)2003 Brown University 
In approaching the problem we shall employ the extended Stroh formalism for piezoelectrics (see Barnett and Lothe 1975, Lothe and Barnett 1976. Suo et al. 1992. Dascalu and Maugin 1995, Chung and Ting 1996), which has been proved to be an elegant and powerful tool in solving two-dimensional problems for anisotropic materials (Ting 1996). Each complex function involved in the general solution for the external domain will be represented as a superposition of complex logarithmic singularities spread along a line segment located inside the lamellar region. A general form of the complex functions entering in the representation of the solution inside the thin region will be deduced as asymptotic expansions around their values on the body axis. By imposing the continuity of the displacements and tractions across the interface curve we obtain a system of integral equations for determining the fields in the lamellar region and the density of the singularities for the external solution. The use of sum rules and identities for the matrices involved in these equations allow us to convert the complex-form system into a real one. The resulting system of integral equations is solved asymptotically, for a small thickness ratio $\varepsilon$ of the lamellar region, by using the technique developed by Geer and Keller (1968) and Homentcovschi (1982) for some plane potential problems. The main advantage of this approach is that it provides a uniform valid asymptotic expansion of the solution which assures the proper behavior of the physical quantities at the body's ends. This technique was applied to plane elasticity problems for isotropic media by Homentcovschi (1984) and Homentcovschi and Dascalu (2000). Asymptotic solutions were also obtained by Wu (1994), using some different perturbation technique.

The method was extended to the case of anisotropic elastic materials by Dascalu and Homentcovschi (1999). This technique requires the distribution of singularities for the external solution on three different segments inside the lamellar region. For piezoelectric materials, considered in this paper, to obtain a uniform valid asymptotic solution, we shall look for the external solution as generated by point sources spread along four different line segments in the lamellar body. This special combination of the Stroh formalism with the above-mentioned technique for solving the corresponding integral equations enables us to obtain the asymptotic expansion of the solution when the inner domain consists of a piezoelectric medium, a rigid and electrically-conducting medium, or a cavity. This later case will be modeled either as an electrically impermeable hole without inside clectric ficlds or as a hole into which electric fields are allowed.

To make the reading of the paper easier, we give only the first two terms in the asymptotic expansion of the solution. Subsequent terms can be obtained by following the outlined procedure. We also give real-form expressions of the elastic and electric fields at the common boundary of the two bodies and on the $O x_{1}$-axis, outside the lamellar inhomogeneity.

As concrete examples, we consider the case of an elliptic lamellar region, corresponding to finite curvature radius at the ends, and also the case of a lemon-shaped inhomogeneity: when we have a zero radius of curvature at the ends of the body. While in the case of an elliptic region the solution was already given in the literature, the results for a lemonshaped inhomogeneity seem to be new. It is found that. while for elliptic piezoelectric inclusions the perturbation stresses and electric displacements at the inclusion ends have the same order as the fields given at infinity. for a lemon-shaped inclusion they are an 
order-of-magnitude smaller. This fact has important consequences for the mechanical resistance of the composite structure.

We also consider cavities with electrically impermeable boundaries and cavities with inside electric fields. Intensity factors are calculated for lemon-shaped cavities. It is observed that, when inside electric fields are considered, the anisotropy influences the stress intensity coefficients. Such an influence was experimentally observed (e.g., Pisarenko et al. 1985), but not predicted by the classical crack models. The obtained result shows the finite-thickness cavities with cusps are more realistic than the classical line models for cracks.

2. Basic Equations. Consider an infinitely extended piezoelectric body $\mathcal{D}^{e}$ that contains an inhomogeneity $\mathcal{D}^{*}$, as shown in Fig. 1. We assume that the interface boundary $\mathcal{C}$ is described by the equations

$$
z_{ \pm}=x_{1}+i \varepsilon Y_{ \pm}\left(x_{1}\right) ; \quad Y_{ \pm}\left(x_{1}\right)=S\left(x_{1}\right) \pm \sqrt{D\left(x_{1}\right)},
$$

where $S\left(x_{1}\right)$ and $D\left(x_{1}\right)$ are twice differentiable functions on the interval $[-1,1]$, satisfying $\max \left|S\left(x_{1}\right) \pm \sqrt{D\left(x_{1}\right)}\right|=1$ and vanishing at the cndpoints \pm 1 . It is also assumed that their behavior about the endpoints can be described by

$$
\begin{aligned}
& S\left(x_{1}\right)=\sum_{n=1}^{2} S_{n}\left(1-x_{1}\right)^{n}+O\left(\left(1-x_{1}\right)^{3}\right) \\
& S\left(x_{1}\right)=\sum_{n=1}^{2} \widetilde{S}_{n}\left(1+x_{1}\right)^{n}+O\left(\left(1+x_{1}\right)^{3}\right)
\end{aligned}
$$

and similarly for $D\left(x_{1}\right)$. Remark that for $D_{1} \neq 0 \neq \widetilde{D}_{1}$, the radius of curvature at each end of the body is nonzero, while for $\widetilde{D}_{1}=\widetilde{D}_{2}=D_{1}=D_{2}=0$, the extremities are cusps.

Let $C_{i j k l}^{e}, e_{l i j}^{e}, \varepsilon_{i l}^{e}$, be, respectively, the elastic, piezoelectric and dielectric constants of the exterior medium $\mathcal{D}^{e}$ and $C_{i j k l}^{*}, e_{l i j}^{*}, \varepsilon_{i l}^{*}$ the corresponding coefficients for the inside body $\mathcal{D}^{*}$. The elastic and dielectric tensors for both media are assumed to be fully symmetric and positive definite. In the absence of body forces and frce charges, the equilibrium equations can be written as (Ticrsten 1969, Maugin 1988)

$$
\begin{aligned}
C_{i j k l}^{e} \frac{\partial^{2} u_{k}^{e}}{\partial x_{l} \partial x_{j}}+e_{l i j}^{e} \frac{\partial^{2} \varphi^{e}}{\partial x_{l} \partial x_{j}}=0 \\
e_{i k l}^{e} \frac{\partial^{2} u_{k}^{e}}{\partial x_{l} \partial x_{i}}-\varepsilon_{i l}^{e} \frac{\partial^{2} \varphi^{e}}{\partial x_{l} \partial x_{i}}=0,
\end{aligned}
$$

where repeated indices imply summation. Here $u_{k}^{e}, k=1,2,3$ and $\varphi^{e}$ are, respectively, the elastic displacements and the electric potential in the matrix $\mathcal{D}^{e}$ and they are supposed to depend on $x_{1}$ and $x_{2}$ only. Let $\epsilon_{i j}^{e}=\frac{1}{2}\left(\frac{\partial u_{i}^{e}}{\partial x_{j}}+\frac{\partial u_{j}^{e}}{\partial x i}\right)$ be the strain tensor and $E_{i}^{e}=-\frac{\partial \varphi^{e}}{\partial x_{1}}$ the electric field. Then the constitutive relations for piezoelectric materials can be written as

$$
\sigma_{i j}^{e}=C_{i j k l} \epsilon_{k l}-e_{l i j}^{e} E_{l}^{e} ; \quad D_{i}^{e}=e_{i j l}^{e} \epsilon_{j l}+\varepsilon_{i l}^{e} E_{l}^{e},
$$


where $\sigma_{i j}^{e}$ is the elastic stress and $D_{i}^{e}$ is the electric displacement. Similar relations have to be considered for the medium inside the inclusion $\mathcal{D}^{*}$.

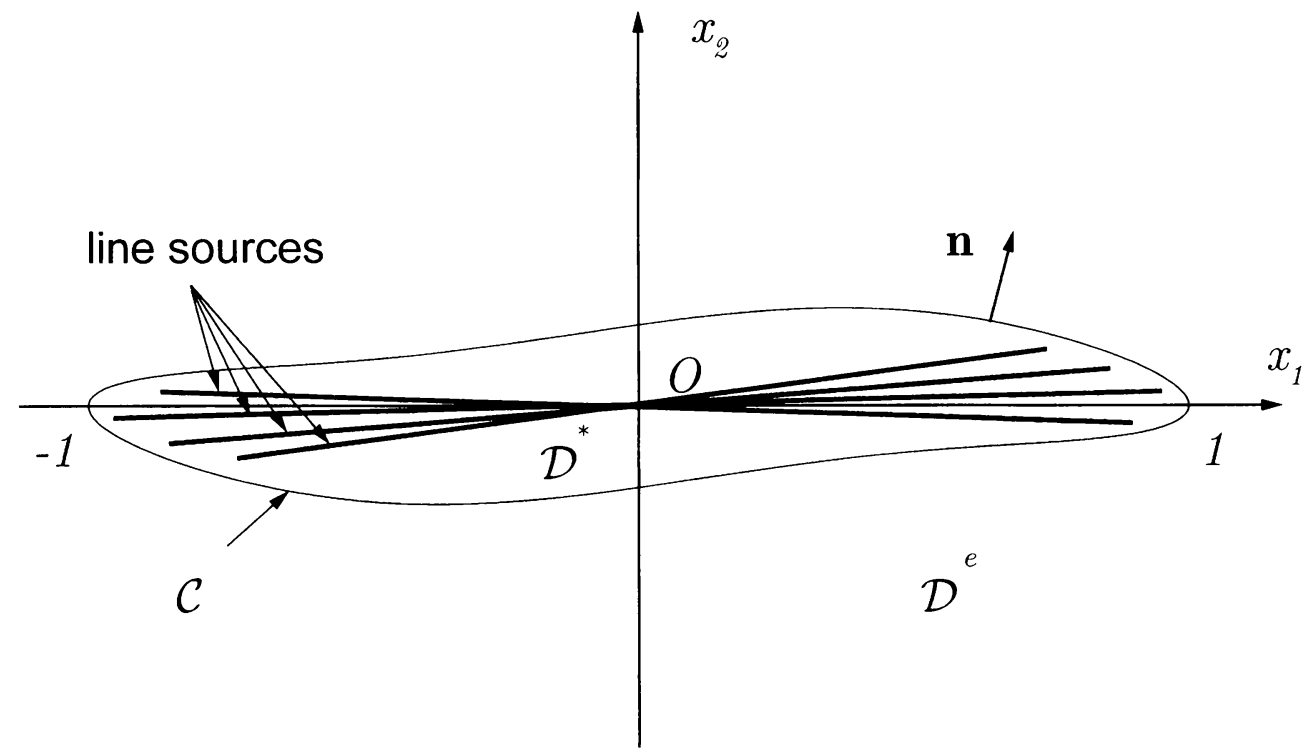

FIG. 1. Lamellar inhomogeneity in an infinite piezoelectric body

We assume that the two bodies have a perfect bonding along their common interface, which means that the elastic displacements, the surface tractions, the electric potential, and the normal electric displacements are continuous:

$$
u_{k}^{*}=u_{k}^{e} ; \quad \boldsymbol{\sigma}^{*} \mathbf{n}=\boldsymbol{\sigma}^{e} \mathbf{n} ; \quad \varphi^{*}=\varphi^{e} ; \quad \mathbf{D}^{*} \mathbf{n}=\mathbf{D}^{e} \mathbf{n},
$$

where $\mathbf{n}$ is the unit normal vector on $\mathcal{C}$ directed toward $\mathcal{D}^{e}$ from $\mathcal{D}^{*}$ (see Fig. 1).

It can be shown (Barnett and Lothe 1975, Chung and Ting 1996) that the stresses and the electric displacements can be expressed in terms of a four-dimensional generalized stress function vector $\phi^{e}$ by

$$
\sigma_{i 1}^{e}=-\frac{\partial \phi_{i}^{e}}{\partial x_{2}} ; \quad \sigma_{i 2}^{e}=\frac{\partial \phi_{i}^{e}}{\partial x_{1}} ; \quad D_{1}^{e}=-\frac{\partial \phi_{4}^{e}}{\partial x_{2}} ; \quad D_{2}^{e}=\frac{\partial \phi_{4}^{e}}{\partial x_{1}} .
$$

If we also define a generalized displacement vector $\mathbf{u}^{e}=\left(u_{1}^{e}, u_{2}^{e}, u_{3}^{e}, \varphi^{e}\right)$, then the perfect bonding conditions in (7) become

$$
\mathbf{u}^{*}=\mathbf{u}^{e} ; \quad \phi^{*}=\phi^{e} \text { on } \mathcal{C} .
$$

We suppose that a uniform stress $\boldsymbol{\sigma}^{e}=\boldsymbol{\sigma}^{\infty}$ and electric displacements $\mathbf{D}^{e}=\mathbf{D}^{\infty}$ are applied at infinity. Let

$$
\mathbf{u}^{\infty}=x_{1} \boldsymbol{\gamma}_{1}^{\infty}+x_{2} \gamma_{2}^{\infty} ; \quad \phi^{\infty}=x_{1} \mathbf{t}_{2}^{\infty}-x_{2} \mathbf{t}_{1}^{\infty},
$$

where

$$
\begin{array}{ll}
\gamma_{1}^{\infty}=\left(\varepsilon_{11}^{\infty}, 0.2 \varepsilon_{13}^{\infty},-E_{1}^{\infty}\right) ; & \gamma_{2}^{\infty}=\left(2 \epsilon_{21}^{\infty}, \epsilon_{22}^{\infty}, 2 \epsilon_{23}^{\infty},-E_{2}^{\infty}\right) \\
\mathbf{t}_{1}^{\infty}=\left(\sigma_{11}^{\infty}, \sigma_{12}^{\infty}, \sigma_{13}^{\infty}, D_{1}^{\infty}\right) ; & \mathbf{t}_{2}^{\infty}=\left(\sigma_{21}^{\infty}, \sigma_{22}^{\infty}, \sigma_{23}^{\infty}, D_{2}^{\infty}\right)
\end{array}
$$


and define

$$
\mathbf{u}=\mathbf{u}^{e}-\mathbf{u}^{\infty} ; \quad \phi=\phi^{e}-\phi^{\infty} .
$$

Then the general solution of our problem has the form (Barnett and Lothe 1975, Chung and Ting 1996):

$$
\begin{aligned}
\mathbf{u}(z) & =2 \operatorname{Re}(\mathbf{A F}(\mathbf{Z})) ; & \boldsymbol{\phi}(z) & =2 \operatorname{Re}(\mathbf{B F}(\mathbf{Z})) \\
\mathbf{u}^{*}(z) & =2 \operatorname{Re}\left(\mathbf{A}^{*} \mathbf{F}^{*}\left(\mathbf{Z}^{*}\right)\right) ; & \boldsymbol{\phi}^{*}(z) & =2 \operatorname{Re}\left(\mathbf{B}^{*} \mathbf{F}^{*}\left(\mathbf{Z}^{*}\right)\right)
\end{aligned}
$$

with

$$
\mathbf{F}(\mathbf{Z})=\left(F_{1}\left(z_{1}\right), F_{2}\left(z_{2}\right), F_{3}\left(z_{3}\right), F_{4}\left(z_{4}\right)\right) ; \quad \mathbf{Z}=\left(z_{1}, z_{2}, z_{3}, z_{4}\right)
$$

and where $z=x_{1}+i x_{2}, z_{\alpha}=x_{1}+p_{\alpha} x_{2}, \alpha=1,2,3,4$. The complex numbers $p_{\alpha}$ are the eigenvalues with positive imaginary parts and $A_{k \alpha}$ are the eigenvectors of the problem:

$$
\left(Q_{l k}+p_{\alpha}\left(R_{l k}+R_{k l}\right)+p_{\alpha}^{2} T_{l k}\right) A_{k \alpha}=0
$$

without summation on $\alpha$ and where the matrices

$$
\begin{gathered}
\mathbf{Q}=\left[\begin{array}{cc}
\mathbf{Q}^{E} & \mathbf{e}_{11} \\
\mathbf{e}_{11}^{T} & -\varepsilon_{11}^{e}
\end{array}\right], \quad \mathbf{R}=\left[\begin{array}{cc}
\mathbf{R}^{E} & \mathbf{e}_{21} \\
\mathbf{e}_{12}^{T} & -\varepsilon_{12}^{e}
\end{array}\right], \quad \mathbf{T}=\left[\begin{array}{cc}
\mathbf{T}^{E} & \mathbf{e}_{22} \\
\mathbf{e}_{22}^{T} & -\varepsilon_{22}^{e}
\end{array}\right] \\
\left(\mathbf{Q}^{E}\right)_{\alpha k}=C_{\alpha 1 k 1}^{e}, \quad\left(\mathbf{R}^{E}\right)_{\alpha k}=C_{\alpha 1 k 2}^{e}, \quad\left(\mathbf{T}^{E}\right)_{\alpha k}=C_{\alpha 2 k 2}^{e}, \quad\left(\mathbf{e}_{i j}\right)_{k}=e_{i j k}^{e} .
\end{gathered}
$$

We assume that the eigenvalues $p_{\alpha}$ are all distinct. The matrix $\mathbf{B}$ is given by

$$
B_{i \alpha}=R_{k i} A_{k \alpha}+p_{\alpha} T_{i k} A_{k \alpha} .
$$

The following closure relations hold true (e.g., Chung and Ting 1996):

$$
\begin{aligned}
& \mathbf{A}^{T} \mathbf{B}+\mathbf{B}^{T} \mathbf{A}=\mathbf{I}=\overline{\mathbf{A}}^{T} \overline{\mathbf{B}}+\overline{\mathbf{B}}^{T} \overline{\mathbf{A}} \\
& \mathbf{A}^{T} \overline{\mathbf{B}}+\mathbf{B}^{T} \overline{\mathbf{A}}=\mathbf{0}=\overline{\mathbf{A}}^{T} \mathbf{B}+\overline{\mathbf{B}}^{T} \mathbf{A} \\
& \mathbf{A} \mathbf{A}^{T}+\overline{\mathbf{A A}}^{T}=\mathbf{0}=\mathbf{B B}^{T}+\overline{\mathbf{B B}}^{T} \\
& \mathbf{B} \mathbf{A}^{T}+\overline{\mathbf{B A}}^{T}=\mathbf{I}=\mathbf{A} \mathbf{B}^{T}+\overline{\mathbf{A B}}^{T} .
\end{aligned}
$$

The corresponding quantities $\mathbf{F}^{*}\left(\mathbf{Z}^{*}\right), \mathbf{Z}^{*}, z_{\alpha}^{*}, p_{\alpha}^{*}, A_{i \alpha}^{*}, B_{i \alpha}^{*}$ are defined in a similar way.

We also introduce the vectorial notation

$$
\frac{d \mathbf{F}(\mathbf{Z})}{d \mathbf{Z}}=\left(\frac{d F_{1}}{d z_{1}}, \frac{d F_{2}}{d z_{2}}, \frac{d F_{3}}{d z_{3}}, \frac{d F_{4}}{d z_{4}}\right)
$$

and the stress-like vectors

$$
\mathbf{t}_{1}=\left(\sigma_{11}, \sigma_{12}, \sigma_{13}, D_{1}\right) ; \quad \mathbf{t}_{2}=\left(\sigma_{21}, \sigma_{22}, \sigma_{23}, D_{2}\right) .
$$

Then, from the representation (14) we have

$$
\mathbf{t}_{1}=-\frac{\partial \phi}{\partial x_{2}}=-2 \operatorname{Re}\left(\mathbf{B P} \frac{d \mathbf{F}}{d \mathbf{Z}}\right) ; \quad t_{2}=\frac{\partial \phi}{\partial x_{1}}=2 \operatorname{Re}\left(\mathbf{B} \frac{d \mathbf{F}}{d \mathbf{Z}}\right),
$$

where $\mathbf{P}$ is the diagonal matrix

$$
\mathbf{P}=\operatorname{diag}\left[p_{1}, p_{2}, p_{3}, p_{4}\right] .
$$


By substituting $\mathbf{u}, \mathbf{u}^{*}, \phi$ and $\phi^{*}$ from $(14-15)$ in the interface relations (9) and by using (13), we obtain

$$
\begin{aligned}
& \mathbf{u}^{\infty}(z)+2 \operatorname{Re}(\mathbf{A F}(\mathbf{Z}))=2 \operatorname{Re}\left(\mathbf{A}^{*} \mathbf{F}^{*}\left(\mathbf{Z}^{*}\right)\right) \\
& \phi^{\infty}(z)+2 \operatorname{Re}(\mathbf{B F}(\mathbf{Z}))=2 \operatorname{Re}\left(\mathbf{B}^{*} \mathbf{F}^{*}\left(\mathbf{Z}^{*}\right)\right)
\end{aligned}
$$

for $z \in \mathcal{C}$. These equations will be studied in the following section.

3. Piezoelectric Inclusions. In order to solve the equations (29-30) we look for $F_{\alpha}\left(z_{\alpha}\right)$ in the form

$$
\begin{aligned}
F_{\alpha}\left(z_{\alpha}\right) & =-\frac{1}{\pi i} \int_{c_{\alpha}}^{d_{\alpha}} \frac{\tilde{f}_{\alpha}(\xi)}{\sqrt{\left(d_{\alpha}-\xi\right)\left(\xi-c_{\alpha}\right)}} \ln \left(z_{\alpha}-\xi\right) d \xi \\
& =-\frac{1}{\pi i} \int_{-1}^{1} \frac{f_{\alpha}(t)}{\sqrt{1-t^{2}}} \ln \left(z_{\alpha}-\frac{c_{\alpha}+d_{\alpha}}{2}-\frac{d_{\alpha}-c_{\alpha}}{2} t\right) d t,
\end{aligned}
$$

with $c_{\alpha}, d_{\alpha} \in \mathbb{C}$ belonging to $\mathcal{D}^{*}$. Note that each potential function $F_{\alpha}$ will be given by a different distribution of line sources (see Fig. 1). From the asymptotic development of $F_{\alpha}\left(z_{\alpha}\right)$ we have deduced in Appendix A the formulae

$$
\begin{aligned}
& \frac{1}{2}\left(F_{\alpha}\left(z_{\alpha+}\right)+F_{\alpha}\left(z_{\alpha-}\right)\right)=\mathbb{K}_{0} f_{\alpha}\left(x_{1}\right)+\varepsilon p_{\alpha} \mathbb{K}_{1} f_{\alpha}\left(x_{1}\right)+O\left(\varepsilon^{2}\right) \\
& \frac{1}{2}\left(F_{\alpha}\left(z_{\alpha+}\right)-F_{\alpha}\left(z_{\alpha-}\right)\right)=\mathbb{L}_{0} f_{\alpha}\left(x_{1}\right)+\varepsilon p_{\alpha} \mathbb{L}_{1} f_{\alpha}\left(x_{1}\right)+O\left(\varepsilon^{2}\right)
\end{aligned}
$$

for $z_{\alpha \pm}=x_{1}+p_{\alpha} \varepsilon Y_{ \pm}\left(x_{1}\right)$ and $x_{1} \in(-1,1)$. In these relations we have $f_{\alpha}=f_{\alpha}\left(x_{1}, \varepsilon\right)$ and we develop the density functions $f_{\alpha}$ as

$$
f_{\alpha}\left(x_{1}, \varepsilon\right)=f_{\alpha}^{0}\left(x_{1}\right)+\varepsilon f_{\alpha}^{1}\left(x_{1}\right)+O\left(\varepsilon^{2}\right) .
$$

In what concerns the solution in the interior domain $\mathcal{D}^{*}$ we use a Taylor expansion about $\varepsilon=0$ :

$$
F_{\alpha}^{*}\left(z_{\alpha \pm}\right)=f_{\alpha}^{*}\left(x_{1}\right)+p_{\alpha}^{*} \varepsilon Y_{ \pm}\left(x_{1}\right) f_{\alpha}^{*(1)}\left(x_{1}\right)+O\left(\varepsilon^{2}\right)
$$

where $f_{\alpha}^{*(1)} \equiv \frac{d f_{\alpha}^{*}}{d x_{1}}$. Similarly, we set

$$
f_{\alpha}^{*}\left(x_{1}, \varepsilon\right)=f_{\alpha}^{(0 *}\left(x_{1}\right)+\varepsilon f_{\alpha}^{1 *}\left(x_{1}\right)+O\left(\varepsilon^{2}\right) .
$$

These asymptotic expansions will be used in the equations (29-30). Before that, we introduce the following real densities, written in vectorial form:

$$
\begin{aligned}
\mathbf{u}^{n}\left(x_{1}\right) & =2 \operatorname{Re}\left(\mathbf{A f}^{n}\left(x_{1}\right)\right) ; & \phi^{n}\left(x_{1}\right) & =2 \operatorname{Re}\left(\mathbf{B} \mathbf{f}^{n}\left(x_{1}\right)\right) \\
\mathbf{u}^{n *}\left(x_{1}\right) & =2 \operatorname{Re}\left(\mathbf{A}^{*} \mathbf{f}^{n *}\left(x_{1}\right)\right) ; & \phi^{n *}\left(x_{1}\right) & =2 \operatorname{Re}\left(\mathbf{B}^{*} \mathbf{f}^{n *}\left(x_{1}\right)\right)
\end{aligned}
$$

for $n=0,1$. The properties $(21-22)$ of the matrices $\mathbf{A}$ and $\mathbf{B}$ cnable us to express the densities $\mathbf{f}^{n}$ and $\mathbf{f}^{n *}$ as

$$
\mathbf{f}^{n}=\mathbf{B}^{T} \mathbf{u}^{n}+\mathbf{A}^{T} \phi^{n} ; \quad \mathbf{f}^{n *}=\mathbf{B}^{* T} \mathbf{u}^{n *}+\mathbf{A}^{* T} \boldsymbol{\phi}^{n *} .
$$

Let us separate the real and imaginary parts of the operators $\mathbb{K}_{m}$ and $\mathbb{L}_{m}$ :

$$
\mathbb{K}_{m}=\widehat{\mathbb{K}}_{m}+i \tilde{\mathbb{K}}_{m} ; \quad \mathbb{L}_{m}=\widehat{\mathbb{L}}_{m}+i \tilde{\mathbb{L}}_{m}
$$

From their definitions (191). (194) we remark that $\mathbb{K}_{0}=i \widetilde{\mathbb{K}}_{0}$ and $\mathbb{L}_{0}=\widehat{\mathbb{L}}_{0}$. 
By addition and subtraction of the equations (29-30) and by use of the expansions $(32-36)$ and the relations (37-39), after identification of the coefficients of each power of $\varepsilon$, we obtain equations for the real densities $\mathbf{u}^{n}, \phi^{n}, \mathbf{u}^{n *}$ and $\phi^{n *}$.

Using the identities (21--24) we find the equations of $O(1)$ as

$$
\begin{aligned}
\gamma_{1}^{\infty} x_{1}+\widetilde{\mathbb{K}}_{0}\left(\mathbf{S u}^{0}\left(x_{1}\right)+\mathbf{H} \phi^{0}\left(x_{1}\right)\right) & =\mathbf{u}^{0 *}\left(x_{1}\right) \\
\widehat{\mathbb{L}}_{0} \mathbf{u}^{0}\left(x_{1}\right) & =\mathbf{0} \\
\mathbf{t}_{2}^{\infty} x_{1}+\widetilde{\mathbb{K}}_{0}\left(-\mathbf{L u}^{0}\left(x_{1}\right)+\mathbf{S}^{T} \phi^{0}\left(x_{1}\right)\right) & =\phi^{0 *}\left(x_{1}\right) \\
\widehat{\mathbb{L}}_{0} \phi^{0}\left(x_{1}\right) & =\mathbf{0}
\end{aligned}
$$

for $x_{1} \in(-1,1)$. The matrices $\mathbf{S}, \mathbf{H}$, and $\mathbf{L}$, first introduced by Barnett and Lothe (1975), are given by

$$
\mathbf{S}=i\left(2 \mathbf{A B}^{T}-\mathbf{I}\right) ; \quad \mathbf{H}=2 i \mathbf{A A}^{T} ; \quad \mathbf{L}=-2 i \mathbf{B B}^{T}
$$

From (23-24) it results that they are real and that $\mathbf{H}$ and $\mathbf{L}$ are symmetric. Lothe and Barnett (1976) showed that they are nonsingular.

The solutions of (41-44) are

$$
\begin{aligned}
\mathbf{u}^{0}\left(x_{1}\right) & =\mathbf{0} ; \quad \phi^{0}\left(x_{1}\right)=\mathbf{0} \\
\mathbf{u}^{0 *}\left(x_{1}\right) & =\gamma_{1}^{\infty} x_{1} ; \quad \phi^{0 *}\left(x_{1}\right)=\mathbf{t}_{2}^{\infty} x_{1} .
\end{aligned}
$$

In order to obtain the higher-order equations we need to introduce the matrices (Chung and Ting 1996):

$$
\mathbf{N}_{1}=-\mathbf{T}^{-1} \mathbf{R}^{T} ; \quad \mathbf{N}_{2}=\mathbf{T}^{-1} ; \quad \mathbf{N}_{3}=\mathbf{R} \mathbf{T}^{-1} \mathbf{R}^{T}-\mathbf{Q} .
$$

The matrices $\mathbf{N}_{2}$ and $\mathbf{N}_{3}$ are symmetric. One can prove (see Ting 1988) the formulae:

$$
\begin{aligned}
2 \mathbf{A P A}^{T} & =\mathbf{N}_{2}-i\left(\mathbf{N}_{1} \mathbf{H}+\mathbf{N}_{2} \mathbf{S}^{T}\right) \\
2 \mathbf{A P B}^{T} & =\mathbf{N}_{1}-i\left(\mathbf{N}_{1} \mathbf{S}-\mathbf{N}_{2} \mathbf{L}\right) \\
2 \mathbf{B P B}^{T} & =\mathbf{N}_{3}-i\left(\mathbf{N}_{3} \mathbf{S}-\mathbf{N}_{1}^{T} \mathbf{L}\right)
\end{aligned}
$$

where $\mathbf{P}$ is the diagonal matrix defined in (28). From (37) and (49-51) we have

$$
\begin{aligned}
2 \operatorname{Re}\left(\mathbb{T} \mathbf{A P f} \mathbf{f}^{m}\right)= & \left(\widehat{\mathbb{T}} \mathbf{N}_{1}+\widetilde{\mathbb{T}}\left(\mathbf{N}_{1} \mathbf{S}-\mathbf{N}_{2} \mathbf{L}\right)\right) \mathbf{u}^{m} \\
& +\left(\widehat{\mathbb{T}} \mathbf{N}_{2}+\widetilde{\mathbb{T}}\left(\mathbf{N}_{1} \mathbf{H}+\mathbf{N}_{2} \mathbf{S}^{T}\right)\right) \boldsymbol{\phi}^{m} \\
2 \operatorname{Re}\left(\mathbb{T} \mathbf{B P f} \mathbf{f}^{m}\right)= & \left(\widehat{\mathbb{T}} \mathbf{N}_{3}+\widetilde{\mathbb{T}}\left(\mathbf{N}_{3} \mathbf{S}-\mathbf{N}_{1}^{T} \mathbf{L}\right)\right) \mathbf{u}^{m} \\
& +\left(\widehat{\mathbb{T}} \mathbf{N}_{1}^{T}+\widetilde{\mathbb{T}}\left(\mathbf{N}_{1}^{T} \mathbf{S}^{T}+\mathbf{N}_{3} \mathbf{H}\right)\right) \boldsymbol{\phi}^{m}
\end{aligned}
$$

for every linear operator $\mathbb{T}=\widehat{\mathbb{T}}+i \widetilde{\mathbb{T}}$. Note that if one replaces $\mathbf{P}=\mathbf{I}$ (identity matrix), $\mathbf{N}_{1}=\mathbf{I}$, and $\mathbf{N}_{2}=\mathbf{N}_{3}=\mathbf{0}$, then (49-53) still hold true. Similar relations can be proved for the interior densities $\mathbf{f}^{m *}, \mathbf{u}^{m *}$, and $\phi^{m *}$. Using these formulae and taking 
into account the solution (46-47), one obtains the system of $O(\varepsilon)$ in real form as:

$$
\begin{gathered}
\gamma_{2}^{\infty} S\left(x_{1}\right)+\frac{1}{\pi} \mathbf{S} \int_{-1}^{1} \frac{\mathbf{u}^{1}(t)}{\sqrt{1-t^{2}}} \ln \left|x_{1}-t\right| d t \\
+\frac{1}{\pi} \mathbf{H} \int_{-1}^{1} \frac{\phi^{1}(t)}{\sqrt{1-t^{2}}} \ln \left|x_{1}-t\right| d t+\mathcal{K}_{1}^{u} \mathbf{u}^{0}\left(x_{1}\right)+\mathcal{K}_{1}^{\phi} \phi^{0}\left(x_{1}\right) \\
=\mathbf{u}^{1 *}\left(x_{1}\right)+\mathcal{K}_{1}^{* u} \mathbf{u}^{* 0}\left(x_{1}\right)+\mathcal{K}_{1}^{* \phi} \phi^{* 0}\left(x_{1}\right) \\
\gamma_{2}^{\infty} \sqrt{D\left(x_{1}\right)}-\int_{x_{1}}^{1} \frac{\mathbf{u}^{1}(t) d t}{\sqrt{1-t^{2}}}+\mathcal{L}_{1}^{u} \mathbf{u}^{0}\left(x_{1}\right)+\mathcal{L}_{1}^{\phi} \phi^{0}\left(x_{1}\right)=\mathcal{L}_{1}^{* u} \mathbf{u}^{* 0}\left(x_{1}\right)+\mathcal{L}_{1}^{* \phi} \boldsymbol{\phi}^{* 0}\left(x_{1}\right) \\
-\mathbf{t}_{1}^{\infty} S\left(x_{1}\right)-\frac{1}{\pi} \mathbf{L} \int_{-1}^{1} \frac{\mathbf{u}^{1}(t)}{\sqrt{1-t^{2}}} \ln \left|x_{1}-t\right| d t \\
+\frac{1}{\pi} \mathbf{S}^{T} \int_{-1}^{1} \frac{\phi^{1}(t)}{\sqrt{1-t^{2}}} \ln \left|x_{1}-t\right| d t+\mathcal{M}_{1}^{u} \mathbf{u}^{0}\left(x_{1}\right)+\mathcal{M}_{1}^{\phi} \phi^{0}\left(x_{1}\right) \\
=\phi^{j *}\left(x_{1}\right)+\mathcal{M}_{1}^{* u} \mathbf{u}^{* 0}\left(x_{1}\right)+\mathcal{M}_{1}^{* \phi} \boldsymbol{\phi}^{* 0}\left(x_{1}\right) \\
-\mathbf{t}_{1}^{\infty} \sqrt{D\left(x_{1}\right)}-\int_{x_{1}}^{1} \frac{\phi^{1}(t) d t}{\sqrt{1-t^{2}}}+\mathcal{N}_{1}^{u} \mathbf{u}^{0}\left(x_{1}\right)+\mathcal{N}_{1}^{\phi} \phi^{0}\left(x_{1}\right) \\
=\mathcal{N}_{1}^{* u} \mathbf{u}^{* 0}\left(x_{1}\right)+\mathcal{N}_{1}^{* \phi} \boldsymbol{\phi}^{* 0}\left(x_{1}\right),
\end{gathered}
$$

where we have introduced the operators

$$
\begin{gathered}
\mathcal{K}_{1}^{u} \mathbf{u}=\left(\widehat{\mathbb{K}}_{1} \mathbf{N}_{1}+\widetilde{\mathbb{K}}_{1}\left(\mathbf{N}_{1} \mathbf{S}-\mathbf{N}_{2} \mathbf{L}\right)\right) \mathbf{u} \\
\mathcal{K}_{1}^{\phi} \boldsymbol{\phi}=\left(\widehat{\mathbb{K}}_{1} \mathbf{N}_{2}+\widetilde{\mathbb{K}}_{1}\left(\mathbf{N}_{1} \mathbf{H}+\mathbf{N}_{2} \mathbf{S}^{T}\right)\right) \boldsymbol{\phi} \\
\mathcal{K}_{1}^{* u} \mathbf{u}=S\left(x_{1}\right) \mathbf{N}_{1}^{*} \mathbf{u} ; \mathcal{K}_{1}^{* \phi} \boldsymbol{\phi}=S\left(x_{1}\right) \mathbf{N}_{2}^{*} \boldsymbol{\phi} \\
\mathcal{L}_{1}^{u} \mathbf{u}=\left(\widehat{\mathbb{L}}_{1} \mathbf{N}_{1}+\widetilde{\mathbb{L}}_{1}\left(\mathbf{N}_{1} \mathbf{S}-\mathbf{N}_{2} \mathbf{L}\right)\right) \mathbf{u} \\
\mathcal{L}_{1}^{\phi} \boldsymbol{\phi}=\left(\widehat{\mathbb{L}}_{1} \mathbf{N}_{2}+\widetilde{\mathbb{L}}_{1}\left(\mathbf{N}_{1} \mathbf{H}+\mathbf{N}_{2} \mathbf{S}^{T}\right)\right) \boldsymbol{\phi} \\
\mathcal{L}_{1}^{* u} \mathbf{u}=\sqrt{D\left(x_{1}\right)} \mathbf{N}_{1}^{*} \mathbf{u} ; \mathcal{L}_{1}^{* \phi} \boldsymbol{\phi}=\sqrt{D\left(x_{1}\right)} \mathbf{N}_{2}^{*} \boldsymbol{\phi} \\
\mathcal{M}_{1}^{u} \mathbf{u}=\left(\widehat{\mathbb{K}}_{1} \mathbf{N}_{3}+\widetilde{\mathbb{K}}_{1}\left(\mathbf{N}_{3} \mathbf{S}-\mathbf{N}_{1}^{T} \mathbf{L}\right)\right) \mathbf{u} \\
\mathcal{M}_{1}^{\phi} \boldsymbol{\phi}=\left(\widehat{\mathbb{K}}_{1} \mathbf{N}_{1}^{T}+\widetilde{\mathbb{K}}_{1}\left(\mathbf{N}_{1}^{T} \mathbf{S}^{T}+\mathbf{N}_{3} \mathbf{H}\right)\right) \boldsymbol{\phi} \\
\mathcal{M}_{1}^{* u} \mathbf{u}=S\left(x_{1}\right) \mathbf{N}_{3}^{*} \mathbf{u} ; \mathcal{M}_{1}^{* \phi} \boldsymbol{\phi}=S\left(x_{1}\right) \mathbf{N}_{1}^{* T} \boldsymbol{\phi} \\
\mathcal{N}_{1}^{u} \mathbf{u}=\left(\widehat{\mathbb{L}}_{1} \mathbf{N}_{3}+\widetilde{\mathbb{L}}_{1}\left(\mathbf{N}_{3} \mathbf{S}-\mathbf{N}_{1}^{T} \mathbf{L}\right)\right) \mathbf{u} \\
\mathcal{N}_{1}^{\phi} \boldsymbol{\phi}=\left(\widehat{\mathbb{L}}_{1} \mathbf{N}_{1}^{T}+\widetilde{\mathbb{L}}_{1}\left(\mathbf{N}_{1}^{T} \mathbf{S}^{T}+\mathbf{N}_{3} \mathbf{H}\right)\right) \boldsymbol{\phi} \\
\mathcal{N}_{1}^{* u} \mathbf{u}=\sqrt{D\left(x_{1}\right)} \mathbf{N}_{3}^{*} \mathbf{u} ; \mathcal{N}_{1}^{* \phi} \boldsymbol{\phi}=\sqrt{D\left(x_{1}\right)} \mathbf{N}_{1}^{* T} \boldsymbol{\phi} .
\end{gathered}
$$


The solution of $O(\varepsilon)$ for the system $(54-57)$ can be obtained as:

$$
\begin{gathered}
\mathbf{u}^{1}\left(x_{1}\right)=-\frac{D^{\prime}\left(x_{1}\right) \sqrt{1-x_{1}^{2}}}{2 \sqrt{D\left(x_{1}\right)}} \mathbf{d}^{1, \infty}, \phi^{1}\left(x_{1}\right)=-\frac{D^{\prime}\left(x_{1}\right) \sqrt{1-x_{1}^{2}}}{2 \sqrt{D\left(x_{1}\right)}} \mathbf{d}^{2, \infty} \\
\mathbf{u}^{* 1}\left(x_{1}\right)=S\left(x_{1}\right) \mathbf{d}^{1, \infty}+\left(\mathbf{S d}^{1, \infty}+\mathbf{H d}^{2, \infty}\right) \frac{1}{\pi} \int_{-1}^{1} \frac{\sqrt{D(t)}}{t-x_{1}} d t \\
\phi^{* 1}\left(x_{1}\right)=S\left(x_{1}\right) \mathbf{d}^{2, \infty}-\left(\mathbf{L} \mathbf{d}^{1, \infty}-\mathbf{S}^{T} \mathbf{d}^{2, \infty}\right) \frac{1}{\pi} \int_{-1}^{1} \frac{\sqrt{D(t)}}{t-x_{1}} d t,
\end{gathered}
$$

where we have introduced

$$
\begin{aligned}
& \mathbf{d}^{1, \infty}=\gamma_{2}^{\infty}-\mathbf{N}_{1}^{*} \gamma_{1}^{\infty}-\mathbf{N}_{2}^{*} \mathbf{t}_{2}^{\infty} \\
& \mathbf{d}^{2, \infty}=-\left(\mathbf{t}_{1}^{\infty}+\mathbf{N}_{3}^{*} \gamma_{1}^{\infty}+\mathbf{N}_{1}^{* T} \mathbf{t}_{2}^{\infty}\right) .
\end{aligned}
$$

We note that systems of equations of $O\left(\varepsilon^{n}\right), n \geq 2$ and their solutions can be obtained in a similar form by employing the same procedure as in the purely elastic case (Dascalu and Homentcovschi 1999), but we restrict to the case $n=0,1$ for the reason of simplicity.

4. Real-form Solutions. The densities $\mathbf{u}^{n}$ and $\phi^{n}$ deduced in the previous section allow us to calculate the solution $\mathbf{u}^{e}, \mathbf{u}^{*}$ of the initial problem (4-5), by making use of (13-15) and (31-39). In this way, the solution will be obtained in a complex-form. In what follows, we give some real-form expressions of the solution.

Let us determine the expressions of generalized displacements and stresses at the interface between the two bodies and on the $O x_{1}$-axis, in the exterior of the inhomogeneity. For the moment, we shall suppose that $\mathbf{u}^{0}\left(x_{1}\right) \neq \mathbf{0}$ and $\phi^{0}\left(x_{1}\right) \neq \mathbf{0}$ so as to obtain general formulae also valid for rigid inclusions and cracks. As we have mentioned before, we neglect the terms of $O\left(\varepsilon^{2}\right)$.

At points on the interface boundary, we have

$$
\mathbf{F}\left(\mathbf{Z}_{ \pm}\right)=\left(\mathbb{N}_{0}^{ \pm} \mathbf{f}^{0}\right)\left(x_{1}\right)+\varepsilon\left(\left(\mathbb{N}_{0}^{ \pm} \mathbf{f}^{1}\right)\left(x_{1}\right)+\left(\mathbb{N}_{1}^{ \pm} \mathbf{P} \mathbf{f}^{0}\right)\left(x_{1}\right)\right)
$$

with $\mathbb{N}_{n}^{ \pm}=\mathbb{K}_{n} \pm \mathbb{L}_{n}$ and $\left(\mathbf{Z}_{ \pm}\right)_{\alpha}=z_{\alpha \pm}$. This formula results from the expansion (195) proved in Appendix A. When it is introduced in the representation (14), we get

$$
\mathbf{u}=2 \operatorname{Re}\left(\mathbb{N}_{0}^{ \pm} \mathbf{A f} \mathbf{f}^{0}\right)+\varepsilon\left(2 \operatorname{Re}\left(\mathbb{N}_{0}^{ \pm} \mathbf{A} \mathbf{f}^{1}+\mathbb{N}_{1}^{ \pm} \mathbf{A} \mathbf{P f} \mathbf{f}^{0}\right)\right) .
$$

Decomposing the operators into real and imaginary parts $\mathbb{N}_{m}^{ \pm}=\widehat{\mathbb{N}}_{m}^{ \pm}+i \widetilde{\mathbb{N}}_{m}^{ \pm}$and using (52) we find

$$
\begin{aligned}
\mathbf{u}=\left[\left(\widehat{\mathbb{N}}_{0}^{ \pm}+\widetilde{\mathbb{N}}_{0}^{ \pm} \mathbf{S}\right) \mathbf{u}^{0}+\widetilde{\mathbb{N}}_{0}^{ \pm} \mathbf{H} \phi^{0}\right]+\varepsilon\left[\left(\widehat{\mathbb{N}}_{0}^{ \pm}+\widetilde{\mathbb{N}}_{0}^{ \pm} \mathbf{S}\right) \mathbf{u}^{1}+\widetilde{\mathbb{N}}_{0}^{ \pm} \mathbf{H} \phi^{1}\right. \\
+\left(\widehat{\mathbb{N}}_{1}^{ \pm} \mathbf{N}_{1}+\widetilde{\mathbb{N}}_{1}^{ \pm}\left(\mathbf{N}_{1} \mathbf{S}-\mathbf{N}_{2} \mathbf{L}\right)\right) \mathbf{u}^{0} \\
\left.\quad+\left(\widehat{\mathbb{N}}_{1}^{ \pm} \mathbf{N}_{2}+\widetilde{\mathbb{N}}_{1}^{ \pm}\left(\mathbf{N}_{1} \mathbf{H}+\mathbf{N}_{2} \mathbf{S}^{T}\right)\right) \phi^{0}\right] .
\end{aligned}
$$

This real-form expression allows us to calculate the elastic displacements and the electric potential at the interface points when the densities $\mathbf{u}^{n}$ and $\phi^{n}$ are known.

Let us obtain similar formulae for stresses. In Appendix A we have proved that

$$
\frac{d \mathbf{F}}{d \mathbf{Z}}\left(\mathbf{Z}_{ \pm}\right)=\left(\mathbb{M}_{0}^{ \pm} \mathbf{f}\right)\left(x_{1}\right)+\varepsilon \mathbf{P}\left(\mathbb{M}_{1}^{ \pm} \mathbf{f}\right)\left(x_{1}\right),
$$


where the operators $\mathbb{M}_{m}^{ \pm}$are given by (199-200). If for $\mathbf{f}$ we make use of the expansion (34), then it results that

$$
\frac{d \mathbf{F}}{d \mathbf{Z}}\left(\mathbf{Z}_{ \pm}\right)=\left(\mathbb{M}_{0}^{ \pm} \mathbf{f}^{0}\right)\left(x_{1}\right)+\varepsilon\left(\left(\mathbb{M}_{0}^{ \pm} \mathbf{f}^{1}+\mathbb{M}_{1}^{ \pm} \mathbf{P f}^{0}\right)\left(x_{1}\right)\right)
$$

Using the relations (27) together with (53) it results

$$
\begin{aligned}
& \mathbf{t}_{1}=- {\left[\left(\widehat{\mathbb{M}}_{0}^{ \pm} \mathbf{N}_{3}+\widetilde{\mathbb{M}}_{0}^{ \pm}\left(\mathbf{N}_{3} \mathbf{S}-\mathbf{N}_{1}^{T} \mathbf{L}\right)\right) \mathbf{u}^{0}+\left(\widehat{\mathbb{M}}_{0}^{ \pm} \mathbf{N}_{1}^{T}+\widetilde{\mathbb{M}}_{0}^{ \pm}\left(\mathbf{N}_{1}^{T} \mathbf{S}^{T}+\mathbf{N}_{3} \mathbf{H}\right)\right) \boldsymbol{\phi}^{0}\right] } \\
&-\varepsilon\left\{\left[\left(\widehat{\mathbb{M}}_{0}^{ \pm} \mathbf{N}_{3}+\widetilde{\mathbb{M}}_{0}^{ \pm}\left(\mathbf{N}_{3} \mathbf{S}-\mathbf{N}_{1}^{T} \mathbf{L}\right)\right) \mathbf{u}^{1}+\left(\widehat{\mathbb{M}}_{0}^{ \pm} \mathbf{N}_{1}^{T}+\widetilde{\mathbb{M}}_{0}^{ \pm}\left(\mathbf{N}_{1}^{T} \mathbf{S}^{T}+\mathbf{N}_{3} \mathbf{H}\right)\right) \phi^{1}\right]\right. \\
&+\left[\left(\widehat{\mathbb{M}}_{1}^{ \pm} \mathbf{N}_{3}^{(2)}+\widetilde{\mathbb{M}}_{1}^{ \pm}\left(\mathbf{N}_{3}^{(2)} \mathbf{S}-\mathbf{N}_{1}^{(2) T} \mathbf{L}\right)\right) \mathbf{u}^{0}\right. \\
&\left.\left.\quad+\left(\widehat{\mathbb{M}}_{1}^{ \pm} \mathbf{N}_{1}^{(2) T}+\widetilde{\mathbb{M}}_{1}^{ \pm}\left(\mathbf{N}_{1}^{(2) T} \mathbf{S}^{T}+\mathbf{N}_{3}^{(2)} \mathbf{H}\right)\right) \boldsymbol{\phi}^{0}\right]\right\} \\
& \mathbf{t}_{2}=-\left[-\widetilde{\mathbb{M}}_{0}^{ \pm} \mathbf{L} \mathbf{u}^{0}+\left(\widehat{\mathbb{M}}_{0}^{ \pm}+\widetilde{\mathbb{M}}_{0}^{ \pm} \mathbf{S}^{T}\right) \phi^{0}\right]-\varepsilon\left\{\left[-\widetilde{\mathbb{M}}_{0}^{ \pm} \mathbf{L} \mathbf{u}^{1}+\left(\widehat{\mathbb{M}}_{0}^{ \pm}+\widetilde{\mathbb{M}}_{0}^{ \pm} \mathbf{S}^{T}\right) \boldsymbol{\phi}^{1}\right]\right. \\
& \quad+\left[\left(\widehat{\mathbb{M}}_{1}^{ \pm} \mathbf{N}_{3}+\widetilde{\mathbb{M}}_{1}^{ \pm}\left(\mathbf{N}_{3} \mathbf{S}-\mathbf{N}_{1}^{T} \mathbf{L}\right)\right) \mathbf{u}^{0}\right. \\
&\left.\left.\quad+\left(\widehat{\mathbb{M}}_{1}^{ \pm} \mathbf{N}_{1}^{T}+\widetilde{\mathbb{M}}_{1}^{ \pm}\left(\mathbf{N}_{1}^{T} \mathbf{S}^{T}+\mathbf{N}_{3} \mathbf{H}\right)\right) \phi^{0}\right]\right\}
\end{aligned}
$$

where the matrices $\mathbf{N}_{1}^{(2)}$ and $\mathbf{N}_{3}^{(2)}$ are defined (see Ting 1988) as

$$
\mathbf{N}_{1}^{(2)}=\mathbf{N}_{1} \mathbf{N}_{1}+\mathbf{N}_{2} \mathbf{N}_{3}, \quad \mathbf{N}_{3}^{(2)}=\mathbf{N}_{3} \mathbf{N}_{1}+\mathbf{N}_{1}^{T} \mathbf{N}_{3}
$$

Next we obtain similar formulae for generalized displacements and stresses on the axis $x_{2}=0$, in the exterior of the inhomogeneity. In Appendix A we give the expansion (196) for $\mathbf{F}\left(x_{1}\right)$ with $\left|x_{1}\right|>1$. If for the densities $f_{\alpha}$ in this expansion we use (34), then it results

$$
\mathbf{F}=\mathbb{R}\left(i \mathbf{f}^{0}\right)+\varepsilon \mathbb{R}\left(i \mathbf{f}^{1}\right),
$$

where the operator $\mathbb{R}$ is defined in (197). By replacing $\mathbf{F}$ in (14) with (83), one obtains

$$
\mathbf{u}=2 \operatorname{Re}\left(i \mathbb{R} \mathbf{A} \mathbf{f}^{0}\right)+\varepsilon 2 \operatorname{Re}\left(i \mathbb{R} \mathbf{A f} \mathbf{f}^{1}\right)
$$

and by (52) we have the real-form displacements

$$
\mathbf{u}=\left[-\widetilde{\mathbb{R}} \mathbf{u}^{0}+\widehat{\mathbb{R}}\left(\mathbf{S} \mathbf{u}^{0}+\mathbf{H} \phi^{0}\right)\right]+\varepsilon\left[-\widetilde{\mathbb{R}} \mathbf{u}^{1}+\widehat{\mathbb{R}}\left(\mathbf{S} \mathbf{u}^{1}+\mathbf{H} \boldsymbol{\phi}^{1}\right)\right] .
$$

Using the expression (202) of $\frac{d \mathbf{F}}{d \mathbf{Z}}$ for $z_{\alpha}=x_{1}$ with $\left|x_{1}\right|>1$, we find the formula (204). Now the stresses are obtained from (27) and (53) as

$$
\begin{gathered}
\mathbf{t}_{1}=-\left[\left(\widetilde{\mathbb{H}} \mathbf{N}_{3}-\widehat{\mathbb{H}}\left(\mathbf{N}_{3} \mathbf{S}-\mathbf{N}_{1}^{T} \mathbf{L}\right)\right) \mathbf{u}^{0}+\left(\widetilde{\mathbb{H}} \mathbf{N}_{1}^{T}-\widehat{\mathbb{H}}\left(\mathbf{N}_{1}^{T} \mathbf{S}^{T}+\mathbf{N}_{3} \mathbf{H}\right)\right) \boldsymbol{\phi}^{0}\right] \\
-\varepsilon\left\{\left[\left(\widetilde{\mathbb{H}} \mathbf{N}_{3}-\widehat{\mathbb{H}}\left(\mathbf{N}_{3} \mathbf{S}-\mathbf{N}_{1}^{T} \mathbf{L}\right)\right) \mathbf{u}^{1}+\left(\widetilde{\mathbb{H}} \mathbf{N}_{1}^{T}-\widehat{\mathbb{H}}\left(\mathbf{N}_{1}^{T} \mathbf{S}^{T}+\mathbf{N}_{3} \mathbf{H}\right)\right) \boldsymbol{\phi}^{1}\right]\right\} \\
\mathbf{t}_{2}=\left[\widehat{\mathbb{H}} \mathbf{L} \mathbf{u}^{0}+\left(\widetilde{\mathbb{H}}-\widehat{\mathbb{H}} \mathbf{S}^{T}\right) \boldsymbol{\phi}^{0}\right]+\varepsilon\left[\widehat{\mathbb{H}} \mathbf{L} \mathbf{u}^{1}+\left(\widetilde{\mathbb{H}}-\widehat{\mathbb{H}} \mathbf{S}^{T}\right) \boldsymbol{\phi}^{1}\right] .
\end{gathered}
$$

In these formulae, the expressions of the real operators $\widehat{\mathbb{H}}$ and $\widetilde{\mathbb{H}}$ can be easily obtained from (203) and (205). We shall give explicit formulae for them in the particular cases considered below.

Note that the physical displacements $\mathbf{u}^{e}$ and the stresses $\mathbf{t}_{i}^{e}, i=1,2$ can be calculated from $\mathbf{u}$ and $\mathbf{t}_{i}$ given before through

$$
\mathbf{u}^{e}=\mathbf{u}^{\infty}+\mathbf{u} ; \quad \mathbf{t}_{i}^{e}=\mathbf{t}_{i}^{\infty}+\mathbf{t}_{i}
$$

as it results from (13). 
Let us explicitly calculate this solution for some specific geometries of the interface boundary $\mathcal{C}$. We first study the problem for a piezoelectric inclusion of elliptic geometry.

The uniform asymptotic solution of this problem is that in (70). For an inclusion of elliptic geometry we have

$$
S\left(x_{1}\right)=0 ; \quad D\left(x_{1}\right)=1-x_{1}^{2}
$$

and by introducing the notation

$$
\begin{gathered}
\widehat{\mathbf{F}}(\mathbf{Z})=\operatorname{diag}\left[\eta_{1}, \eta_{2}, \eta_{3}\right] \\
\eta_{\alpha}=\frac{z_{\alpha}-\sqrt{z_{\alpha}^{2}-\left(1+\frac{p_{\alpha}^{2} \varepsilon^{2}}{2}\right)^{2}}}{1+\frac{p_{\alpha}^{2} \varepsilon^{2}}{2}}
\end{gathered}
$$

the relations $(70),(14),(31),(37)$ give us the solution of $O(\varepsilon)$ :

$$
\begin{aligned}
\mathbf{u} & =-2 \varepsilon \operatorname{Re}\left[i \mathbf{A} \widehat{\mathbf{F}}(\mathbf{Z})\left(\mathbf{B}^{T} \mathbf{d}^{1, \infty}+\mathbf{A}^{T} \mathbf{d}^{2, \infty}\right)\right] \\
\boldsymbol{\phi} & =-2 \varepsilon \operatorname{Re}\left[i \mathbf{B} \widehat{\mathbf{F}}(\mathbf{Z})\left(\mathbf{B}^{T} \mathbf{d}^{1, \infty}+\mathbf{A}^{T} \mathbf{d}^{2, \infty}\right)\right]
\end{aligned}
$$

where $\mathbf{d}^{1, \infty}$ and $\mathbf{d}^{2, \infty}$ are defined in (73-74).

In the interior of the inclusion, the approximation of $O(\varepsilon)$ of the uniform asymptotic solution is provided by the formulae

$$
\begin{aligned}
& \mathbf{u}^{*}=\mathbf{u}^{0 *}+\varepsilon \mathbf{u}^{1 *}+x_{2}\left(\mathbf{N}_{1}^{*} \mathbf{u}^{0 *(1)}+\mathbf{N}_{2}^{*} \phi^{0 *(1)}\right) \\
& \phi^{*}=\phi^{0 *}+\varepsilon \phi^{1 *}+x_{2}\left(\mathbf{N}_{3}^{*} \mathbf{u}^{0 *(1)}+\mathbf{N}_{1}^{* T} \phi^{0 *(1)}\right),
\end{aligned}
$$

in which $\mathbf{u}^{0 *}$ and $\phi^{0 *}$ are given by (47), while $\mathbf{u}^{1 *}$ and $\phi^{1 *}$ are obtained from (71-72) in the form

$$
\mathbf{u}^{1 *}=-\left(\mathbf{S d}^{1, \infty}+\mathbf{H d}^{2, \infty}\right) x_{1} ; \quad \phi^{1 *}=\left(\mathbf{L d}^{1, \infty}-\mathbf{S}^{T} \mathbf{d}^{2, \infty}\right) x_{1}
$$

By substituting $\mathbf{u}^{1 *}$ and $\phi^{1 *}$ in (94-95) one obtains the same expressions as in Liang et al. (1995), Chung and Ting (1996). That is, in the approximation of $O(\varepsilon)$, the two solutions are identical. This is also true for the exterior solution (92-93).

When (89) are used in the general solutions (70-72) we find

$$
\mathbf{u}^{1}\left(x_{1}\right)=x_{1} \mathbf{d}^{1, \infty} ; \quad \phi^{1}\left(x_{1}\right)=x_{1} \mathbf{d}^{2, \infty} .
$$

These solutions substituted into (80-81) provide the expressions of the interface generalized stresses

$$
\begin{aligned}
\mathbf{t}_{1}=- & -\varepsilon x_{1}\left[\left(\mathbf{N}_{3} \mathbf{d}^{1, \infty}+\mathbf{N}_{1}^{T} \mathbf{d}^{2, \infty}\right) \hat{h}^{ \pm}\left(x_{1}\right)\right. \\
& \left.+\left(\left(\mathbf{N}_{3} \mathbf{S}-\mathbf{N}_{1}^{T} \mathbf{L}\right) \mathbf{d}^{1, \infty}+\left(\mathbf{N}_{1}^{T} \mathbf{S}^{T}+\mathbf{N}_{3} \mathbf{H}\right) \mathbf{d}^{2, \infty}\right) \tilde{h}^{ \pm}\left(x_{1}\right)\right] \\
\mathbf{t}_{2}=- & \varepsilon x_{1}\left[\hat{h}^{ \pm}\left(x_{1}\right)-\left(\mathbf{L} \mathbf{d}^{1, \infty}-\mathbf{S}^{T} \mathbf{d}^{2, \infty}\right) \tilde{h}^{ \pm}\left(x_{1}\right)\right],
\end{aligned}
$$

where $\hat{h}^{ \pm}$and $\tilde{h}^{ \pm}$are the real and the imaginary parts of (201). By introducing

$$
\begin{aligned}
& \mu\left(x_{1}\right)=\arg \left(1+x_{1}\left(1-\frac{\varepsilon^{2} p_{\alpha}^{2}}{2}\right)+\varepsilon p_{\alpha} Y_{ \pm}\right) \\
& \nu\left(x_{1}\right)=\arg \left(1-x_{1}\left(1-\frac{p_{\alpha}^{2} \varepsilon^{2}}{2}\right)-\varepsilon p_{\alpha} Y_{ \pm}\right)
\end{aligned}
$$


with $\mu=0$ and $\nu=0$ for $\varepsilon=0$, we can give completely explicit (98-99). We shall do this for $x_{1}= \pm 1$ (when $\left.Y_{ \pm}=0\right)$. We have

$$
h^{ \pm}( \pm 1)=\frac{\cos \left(\frac{\mu+\nu}{2}( \pm 1)\right)-i \sin \left(\frac{\mu+\nu}{2}( \pm 1)\right)}{\varepsilon \sqrt[4]{\left\{\left[2 \mp \frac{\varepsilon^{2}}{2}\left(\hat{p}_{\alpha}^{2}-\tilde{p}_{\alpha}^{2}\right)\right]^{2}+\varepsilon^{4} \hat{p}_{\alpha}^{2} \tilde{p}_{\alpha}^{2}\right\}\left[\hat{p}_{\alpha}^{2} \tilde{p}_{\alpha}^{2}+\frac{1}{4}\left(\hat{p}_{\alpha}^{2}-\tilde{p}_{\alpha}^{2}\right)^{2}\right.},}
$$

where $\hat{p}_{\alpha}$ and $\tilde{p}_{\alpha}$ stand for the real and, respectively, imaginary parts of $p_{\alpha}$. Substitution into (98-99) together with (88) show that, at the endpoints, the stresses and the electric displacements have the same order (in $\varepsilon$ ) as those given at the infinity. In the purely elastic case, this result was obtained in Homentcovschi and Dascalu (2000) for isotropic materials. For elastic anisotropic materials, the above expressions represent a more refined real-form solution than that given in Dascalu and Homentcovschi (1999), which for the elliptic geometry had not the ability to catch the complete endpoints behavior.

Consider now a lemon-shaped piezoclectric inclusion (see Fig. 2). In this case, the boundary $\mathcal{C}$ is described by

$$
S\left(x_{1}\right)=0 ; \quad D\left(x_{1}\right)=\left(1-x_{1}^{2}\right)^{3} .
$$

For the densities $\mathbf{u}^{0}$ and $\phi^{0}$ the expressions (46-47) hold true, while from (70-72) it results

$$
\begin{gathered}
\mathbf{u}^{1}\left(x_{1}\right)=3 x_{1}\left(1-x_{1}^{2}\right) \mathbf{d}^{1, \infty} ; \quad \phi^{1}\left(x_{1}\right)=3 x_{1}\left(1-x_{1}^{2}\right) \mathbf{d}^{2, \infty} \\
\mathbf{u}^{* 1}\left(x_{1}\right)=\frac{2 x_{1}^{3}-3 x_{1}}{2}\left(\mathbf{S d}^{1, \infty}+\mathbf{H d}^{2, \infty}\right) \\
\boldsymbol{\phi}^{* 1}\left(x_{1}\right)=-\frac{2 x_{1}^{3}-3 x_{1}}{2}\left(\mathbf{L} \mathbf{d}^{1, \infty}-\mathbf{S}^{T} \mathbf{d}^{2, \infty}\right) .
\end{gathered}
$$

With these solutions, we obtain from (77) the displacement-like vectors at the interface

$$
\mathbf{u}\left(x_{1}\right)=\varepsilon\left[\frac{2 x_{1}^{3}-3 x_{1}}{2}\left(\mathbf{S d}^{1, \infty}+\mathbf{H d}^{2, \infty}\right) \mp \sqrt{\left(1-x_{1}^{2}\right)^{3}} \mathbf{d}^{1, \infty}\right]
$$

and from (80-81) the interface generalized stresses

$$
\begin{gathered}
\left.\mathbf{t}_{1}=-\varepsilon\left[\frac{3}{2}\left(2 x_{1}^{2}-1\right)\left(\left(\mathbf{N}_{3} \mathbf{S}-\mathbf{N}_{1}^{T} \mathbf{L}\right)\right) \mathbf{d}^{1, \infty}+\left(\mathbf{N}_{1}^{T} \mathbf{S}^{T}+\mathbf{N}_{3} \mathbf{H}\right)\right) \mathbf{d}^{2, \infty}\right) \\
\left. \pm 3 x_{1} \sqrt{1-x_{1}^{2}}\left(\mathbf{N}_{3} \mathbf{d}^{1, \infty}+\mathbf{N}_{1}^{T} \mathbf{d}^{2, \infty}\right)\right] \\
\left.\mathbf{t}_{2}=\varepsilon\left[\frac{3}{2}\left(2 x_{1}^{2}-1\right)\left(-\mathbf{L} \mathbf{d}^{1, \infty}+\mathbf{S}^{T} \mathbf{d}^{2, \infty}\right) \pm 3 x_{1} \sqrt{1-x_{1}^{2}} \mathbf{d}^{2, \infty}\right)\right]
\end{gathered}
$$

for $\left|x_{1}\right|<1$.

Since the vectors $\mathbf{t}_{1}$ and $\mathbf{t}_{2}$ have physical relevance, it is important to compare the formulae (106-107) with the corresponding ones (98-99) for elliptic inclusions. While in the elliptic case the endpoints' mechanical stresses and electric displacements have the same order as the remote fields, for a lemon-shaped inclusion they are an orderof-magnitude smaller. That is, the lemon-shaped inclusion is more convenient than the elliptic one, from the point of view of the mechanical resistance of the composite structure. 


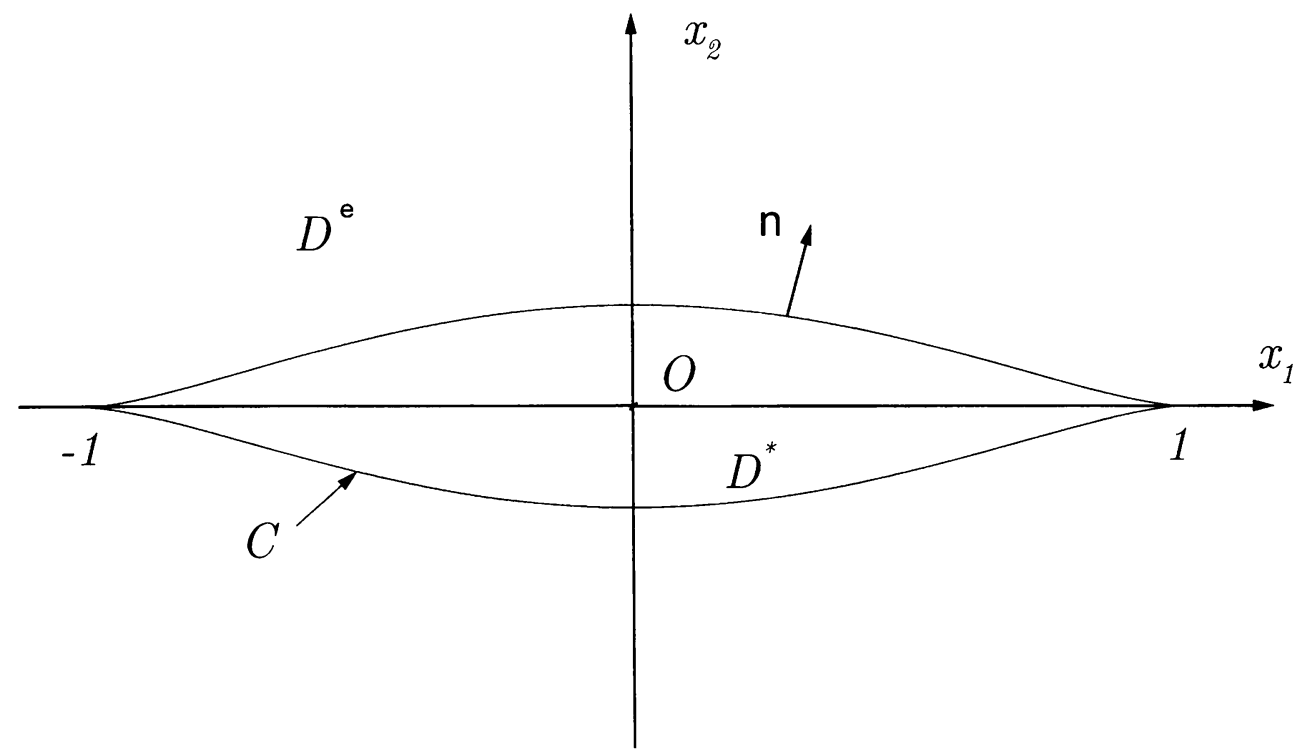

FIG. 2. Lemon-shaped inhomogeneity in a piezoelectric body

Finally, we obtain the generalized displacements and stresses on the axis $O x_{1}$ with $\left|x_{1}\right|>1$, for a lemon-shaped inclusion. The formula (85) leads to

$$
\begin{aligned}
\mathbf{u}\left(x_{1}\right)=\varepsilon\left\{\left[\frac{3}{4}\right.\right. & \left(\operatorname{sgn}\left(x_{1}\right) \sqrt{x_{1}^{2}-1}-x_{1}\right) \\
& \left.\left.-\frac{1}{4}\left(\operatorname{sgn}\left(x_{1}\right) \sqrt{x_{1}^{2}-1}-x_{1}\right)^{3}\right]\left(\mathbf{S d}^{1, \infty}+\mathbf{H d}^{2, \infty}\right)\right\}
\end{aligned}
$$

where $\operatorname{sgn}\left(x_{1}\right)=1$ for $x_{1}>1$ and $\operatorname{sgn}\left(x_{1}\right)=-1$ for $x_{1}<-1$. The stress-like vector functions up to $O(\varepsilon)$ are given by $(86-87)$ as

$$
\begin{aligned}
\mathbf{t}_{1}=\varepsilon & {\left[\frac{3}{2}\left(1-2 x_{1}^{2}+2\left|x_{1}\right| \sqrt{x_{1}^{2}-1}\right)\right.} \\
& \left.\left.\left.\times\left(\left(\mathbf{N}_{3} \mathbf{S}-\mathbf{N}_{1}^{T} \mathbf{L}\right)\right) \mathbf{d}^{1, \infty}+\left(\mathbf{N}_{1}^{T} \mathbf{S}^{T}+\mathbf{N}_{3} \mathbf{H}\right)\right) \mathbf{d}^{2, \infty}\right)\right] \\
\mathbf{t}_{2}=-\varepsilon & {\left[\frac{3}{2}\left(1-2 x_{1}^{2}+2\left|x_{1}\right| \sqrt{x_{1}^{2}-1}\right)\left(-\mathbf{L} \mathbf{d}^{1, \infty}+\mathbf{S}^{T} \mathbf{d}^{2, \infty}\right)\right] . }
\end{aligned}
$$

Note that at the endpoints, the formulae (105-107) and, respectively, (108-110) provide the same fields values.

5. Rigid Inclusions. Consider now the case of a rigid and electrically conducting inclusion $\mathcal{D}^{*}$ embedded in an elastic matrix $\mathcal{D}$, infinitely extended. In such a case we 
have the boundary condition

$$
\begin{gathered}
\mathbf{u}^{\infty}+\mathbf{u}=\omega\left(x_{1} \mathbf{m}-x_{2} \mathbf{n}\right) \\
\mathbf{m}^{T}=(0,1,0,0) ; \quad \mathbf{n}^{T}=(1,0,0,0)
\end{gathered}
$$

on the interface boundary $\mathcal{C}$, where $\omega$ is the rotation of the rigid inclusion. We also impose the condition that the total moment about the origin due to the traction forces on the inclusion boundary vanishes:

$$
\int_{\mathcal{C}}\left(x_{2} \frac{d \phi_{1}}{d s}-x_{1} \frac{d \phi_{2}}{d s}\right) d s=0 .
$$

If in the equation (111) we use the asymptotic expansion

$$
\omega=\omega_{0}+\omega_{1} \varepsilon^{1}+O\left(\varepsilon^{2}\right),
$$

then, by the same procedure as in the previous section, we obtain the system of $O(1)$ under the real form:

$$
\begin{aligned}
\gamma_{1}^{\infty} x_{1}+\widetilde{\mathbb{K}}_{0}\left(\mathbf{S u}^{0}\left(x_{1}\right)+\mathbf{H} \phi^{0}\left(x_{1}\right)\right) & =\omega_{0} x_{1} \mathbf{m} \\
\widehat{\mathbb{L}}_{0} \mathbf{u}^{0}\left(x_{1}\right) & =\mathbf{0} .
\end{aligned}
$$

The solution of these equations is

$$
\mathbf{u}^{0}\left(x_{1}\right)=\mathbf{0} ; \quad \boldsymbol{\phi}^{0}\left(x_{1}\right)=\mathbf{H}^{-1}\left(\boldsymbol{\gamma}_{1}^{\infty}-\omega_{0} \mathbf{m}\right) x_{1}=\phi^{00} x_{1},
$$

where $\phi^{00}$ was introduced in order to simplify the form of the following results.

As for elastic inclusions, the equations of $O(\varepsilon)$ can be written:

$$
\begin{gathered}
\gamma_{2}^{\infty} S\left(x_{1}\right)+\frac{1}{\pi} \mathbf{S} \int_{-1}^{1} \frac{\mathbf{u}^{1}(t)}{\sqrt{1-t^{2}}} \ln \left|x_{1}-t\right| d t \\
+\frac{1}{\pi} \mathbf{H} \int_{-1}^{1} \frac{\phi^{1}(t)}{\sqrt{1-t^{2}}} \ln \left|x_{1}-t\right| d t+\mathcal{K}_{1}^{u} \mathbf{u}^{0}\left(x_{1}\right)+\mathcal{K}_{1}^{\phi} \phi^{0}\left(x_{1}\right) \\
\quad=\omega_{1} x_{1} \mathbf{m}-\omega_{0} S\left(x_{1}\right) \mathbf{n} \\
\gamma_{2}^{\infty} \sqrt{D\left(x_{1}\right)}-\int_{x_{1}}^{1} \frac{\mathbf{u}^{1}(t) d t}{\sqrt{1-t^{2}}}+\mathcal{L}_{1}^{u} \mathbf{u}^{0}\left(x_{1}\right)+\mathcal{L}_{1}^{\phi} \phi^{0}\left(x_{1}\right) \\
=-\omega_{0} \sqrt{D\left(x_{1}\right)} \mathbf{n},
\end{gathered}
$$

with the operators $\mathcal{K}$ and $\mathcal{L}$ defined by (58-59) and, respectively, (61-62). The equations (118-119) have the solutions

$$
\begin{aligned}
& \mathbf{u}^{1}\left(x_{1}\right)=-\sqrt{1-x_{1}^{2}} \frac{d}{d x_{1}}\left\{\gamma_{2}^{\infty} \sqrt{D\left(x_{1}\right)}\right. \\
& \left.+\left(\mathcal{L}_{1}^{u} \mathbf{u}^{0}\left(x_{1}\right)+\mathcal{L}_{1}^{\phi} \phi^{0}\left(x_{1}\right)\right)+\omega_{0} \sqrt{D\left(x_{1}\right)} \mathbf{n}\right\} \\
& \phi^{1}\left(x_{1}\right)=-\omega_{1} \mathbf{H}^{-1} \mathbf{m} x_{1}-\mathbf{H}^{-1} \mathbb{C}^{-1}\left\{S\left(x_{1}\right)\left(\omega_{0} \mathbf{n}+\gamma_{2}^{\infty}\right)\right. \\
& \left.+\left(\mathcal{K}_{1}^{u} \mathbf{u}^{0}\left(x_{1}\right)+\mathcal{K}_{1}^{\phi} \phi^{0}\left(x_{1}\right)\right)\right\}-\mathbf{H}^{-1} \mathbf{S} \mathbf{u}^{1}\left(x_{1}\right),
\end{aligned}
$$


where $\mathbb{C}^{-1}$ is the solution operator of the Carleman integral equation:

$$
\mathbb{C}^{-1} g\left(x_{1}\right)=\frac{1}{\pi} \int_{-1}^{1} \frac{g^{\prime}(t) \sqrt{1-t^{2}}}{t-x_{1}} d t-\frac{1}{\pi \ln 2} \int_{-1}^{1} \frac{g(t)}{\sqrt{1-t^{2}}} d t,
$$

where the prime sign means derivative. For the determination of the constants $\omega_{j}$, we remark that the condition (113) takes the form

$$
\begin{aligned}
\mathbf{m}^{T} \int_{-1}^{1} x_{1}\left(\frac{d \boldsymbol{\phi}_{+}}{d x_{1}}-\frac{d \boldsymbol{\phi}_{-}}{d x_{1}}\right) d x_{1}=\varepsilon \mathbf{n}^{T} & \left\{\int_{-1}^{1} S\left(x_{1}\right)\left(\frac{d \boldsymbol{\phi}_{+}}{d x_{1}}-\frac{d \boldsymbol{\phi}_{-}}{d x_{1}}\right) d x_{1}\right. \\
+ & \left.\int_{-1}^{1} \sqrt{D\left(x_{1}\right)}\left(\frac{d \boldsymbol{\phi}_{+}}{d x_{1}}+\frac{d \boldsymbol{\phi}_{-}}{d x_{1}}\right) d x_{1}\right\} .
\end{aligned}
$$

Let us calculate the expressions in the parentheses. When the expansions (32-34) are replaced in the second relation (14) and the formulae $(52-53)$ are used, one obtains

$$
\begin{aligned}
\left(\frac{d \phi_{+}}{d x_{1}}-\frac{d \phi_{-}}{d x_{1}}\right)\left(x_{1}\right)= & \frac{\phi^{0}\left(x_{1}\right)}{\sqrt{1-x_{1}^{2}}}+\varepsilon\left\{\frac{\phi^{1}\left(x_{1}\right)}{\sqrt{1-x_{1}^{2}}}\right. \\
& \left.\quad+\frac{d}{d x_{1}}\left(\mathcal{N}_{1}^{u} \mathbf{u}^{0}\left(x_{1}\right)+\mathcal{N}_{1}^{\phi} \phi^{0}\left(x_{1}\right)\right)\right\} \\
\left(\frac{d \phi_{+}}{d x_{1}}+\frac{d \phi_{-}}{d x_{1}}\right)\left(x_{1}\right) & =\frac{d}{d x_{1}}\left[\widetilde{\mathbb{K}}_{0}\left(-\mathbf{L} \mathbf{u}^{0}\left(x_{1}\right)+\mathbf{S}^{T} \boldsymbol{\phi}^{0}\left(x_{1}\right)\right)\right] \\
& +\varepsilon \frac{d}{d x_{1}}\left\{\widetilde{\mathbb{K}}_{0}\left(-\mathbf{L} \mathbf{u}^{1}\left(x_{1}\right)+\mathbf{S}^{T} \phi^{1}\left(x_{1}\right)\right)+\mathcal{M}_{1}^{u} \mathbf{u}^{0}\left(x_{1}\right)+\mathcal{M}_{1}^{\phi} \phi^{0}\left(x_{1}\right)\right\}
\end{aligned}
$$

By replacing these expressions in (123), we get

$$
\begin{gathered}
\omega_{0}=\frac{\mathbf{m}^{T} \mathbf{H}^{-1} \boldsymbol{\gamma}_{1}^{\infty}}{\mathbf{m}^{T} \mathbf{H}^{-1} \mathbf{m}} \\
\omega_{1}=\left(\mathbf{m}^{T} \mathbf{H}^{-1} \mathbf{m}\right)^{-1}\left\{\left(\mathbf{m}^{T} \mathbf{H}^{-1} \mathbf{N}_{2}-\mathbf{n}^{T}\right) \phi^{00}-\mathbf{m}^{T} \mathbf{H}^{-1} \mathbf{S} \boldsymbol{\gamma}_{2}^{1, \infty}\right\},
\end{gathered}
$$

where we have introduced the notation

$$
\gamma_{2}^{1, \infty}=\gamma_{2}^{\infty}+\omega_{0} \mathbf{n}-\left(\mathbf{N}_{1} \mathbf{H}+\mathbf{N}_{2} \mathbf{S}^{T}\right) \phi^{00} .
$$

The solution of $O(\varepsilon)$ becomes

$$
\begin{gathered}
\mathbf{u}^{1}\left(x_{1}\right)=-\sqrt{1-x_{1}^{2}}\left\{\frac{D^{\prime}\left(x_{1}\right)}{2 \sqrt{D\left(x_{1}\right)}} \gamma_{2}^{1, \infty}+\frac{d}{d x_{1}}\left(\frac{x_{1} S\left(x_{1}\right)}{\sqrt{1-x_{1}^{2}}}\right) \mathbf{N}_{2} \phi^{00}\right\} \\
\phi^{1}\left(x_{1}\right)=-\omega_{1} \mathbf{H}^{-1} \mathbf{m} x_{1}-\mathbf{H}^{-1} \gamma_{2}^{1, \infty} \mathbb{C}^{-1}\left(S\left(x_{1}\right)\right) \\
-\mathbf{H}^{-1} \mathbf{N}_{2} \phi^{00} \mathbb{C}^{-1}\left(\frac{x_{1} \sqrt{D\left(x_{1}\right)}}{\sqrt{1-x_{1}^{2}}}\right)-\mathbf{H}^{-1} \mathbf{S u}^{1}\left(x_{1}\right) .
\end{gathered}
$$

Consider now the particular case of a lemon-shaped rigid inclusion of electric conductor. The equation of the interface is (102). We have $\mathbf{u}^{0}$ and $\phi^{0}$ given by (117) and (126). 
From (129-130) we get

$$
\begin{gathered}
\mathbf{u}^{1}\left(x_{1}\right)=3 x_{1}\left(1-x_{1}^{2}\right) \boldsymbol{\gamma}_{2}^{1, \infty} \\
\phi^{1}\left(x_{1}\right)=\mathbf{H}^{-1}\left[3 x_{1}^{3}\left(\mathbf{S} \boldsymbol{\gamma}_{2}^{1, \infty}-\mathbf{N}_{2} \phi^{00}\right)-x_{1}\left(\omega_{1} \mathbf{m}-\frac{5}{2} \mathbf{N}_{2} \phi^{00}+3 \mathbf{S} \boldsymbol{\gamma}_{2}^{1, \infty}\right)\right],
\end{gathered}
$$

where $\omega_{1}$ is given by (127). These solutions allow us to calculate the displacements and the electric potential at the interface

$$
\mathbf{u}\left(x_{1}\right)=-\mathbf{H} \phi^{00} x_{1}+\varepsilon\left[\omega_{1} \mathbf{m} x_{1} \mp \sqrt{\left(1-x_{1}^{2}\right)^{3}}\left(\gamma_{2}^{1, \infty}+\left(\mathbf{N}_{2} \mathbf{S}^{T}+\mathbf{N}_{1} \mathbf{H}\right)\right) \phi^{00}\right]
$$

and the interface stresses and electric displacements:

$$
\begin{aligned}
\mathbf{t}_{1}=[ & \left.\left(\mathbf{N}_{1}^{T} \mathbf{S}^{T}+\mathbf{N}_{3} \mathbf{H}\right) \phi^{00} \mp \frac{x_{1}}{\sqrt{1-x_{1}^{2}}} \mathbf{N}_{1}^{T} \phi^{00}\right] \\
& -\varepsilon\left\{3 x_{1}^{2}\left[-\mathbf{N}_{1}^{T} \mathbf{H}^{-1} \boldsymbol{\gamma}_{2}^{1, \infty}+\left(\mathbf{N}_{1}^{T} \mathbf{S}^{T} \mathbf{H}^{-1}+\mathbf{N}_{3}\right) \mathbf{N}_{2} \boldsymbol{\phi}^{00}\right] \pm 3 x_{1} \sqrt{1-x_{1}^{2}} \mathbf{N}_{3} \boldsymbol{\gamma}_{2}^{1, \infty}\right. \\
\mp & \frac{x_{1}}{\sqrt{1-x_{1}^{2}}} \mathbf{N}_{1}^{T} \mathbf{H}^{-1}\left[\left(\omega_{1} \mathbf{m}-\frac{5}{2} \mathbf{N}_{2} \phi^{00}+3 \mathbf{S} \boldsymbol{\gamma}_{2}^{1, \infty}\right)+3 x_{1}^{2}\left(\mathbf{N}_{2} \phi^{00}-\mathbf{S} \boldsymbol{\gamma}_{2}^{1, \infty}\right)\right] \\
+ & \left.\frac{1}{2}\left[3 \mathbf{N}_{1}^{T} \mathbf{H}^{-1} \boldsymbol{\gamma}_{2}^{1, \infty}+\left(\mathbf{N}_{1}^{T} \mathbf{S}^{T} \mathbf{H}^{-1}+\mathbf{N}_{3}\right)\left(\omega_{1} \mathbf{m}-2 \mathbf{N}_{2} \phi^{00}\right)\right]\right\} \\
\mathbf{t}_{2}= & \left(-\mathbf{S}^{T} \phi^{00} \pm \frac{x_{1}}{\sqrt{1-x_{1}^{2}}} \phi^{00}\right)+\varepsilon\left\{-3 x_{1}^{2} \mathbf{H}^{-1}\left(\boldsymbol{\gamma}_{2}^{1, \infty}+\mathbf{S N}_{2} \phi^{00}\right)\right. \\
& \mp \frac{x_{1}}{\sqrt{1-x_{1}^{2}}} \mathbf{H}^{-1}\left[\left(\omega_{1} \mathbf{m}-\frac{5}{2} \mathbf{N}_{2} \phi^{00}+3 \mathbf{S} \gamma_{2}^{1, \infty}\right)+3 x_{1}^{2}\left(\mathbf{N}_{2} \phi^{00}-\mathbf{S} \boldsymbol{\gamma}_{2}^{1, \infty}\right)\right] \\
& \left.+\mathbf{H}^{-1}\left(3 \boldsymbol{\gamma}_{2}^{1, \infty}+2 \mathbf{S N} \boldsymbol{N}_{2} \phi^{00}-2 \omega_{1} \mathbf{S m}\right)\right\} .
\end{aligned}
$$

On the axis $O x_{1},\left|x_{1}\right|>1$, the electric potential and the displacements are

$$
\begin{aligned}
& \mathbf{u}\left(x_{1}\right)=(\left.\operatorname{sgn}\left(x_{1}\right) \sqrt{x_{1}^{2}-1}-x_{1}\right) \mathbf{H} \boldsymbol{\phi}^{00} \\
&+\varepsilon\left\{\frac { 1 } { 4 } \left[\left(\operatorname{sgn}\left(x_{1}\right) \sqrt{x_{1}^{2}-1}-x_{1}\right)\left(\mathbf{N}_{2} \boldsymbol{\phi}^{00}-4 \omega_{1} \mathbf{m}\right)\right.\right. \\
&\left.\left.\quad-\left(\operatorname{sgn}\left(x_{1}\right) \sqrt{x_{1}^{2}-1}-x_{1}\right)^{3} \mathbf{N}_{2} \boldsymbol{\phi}^{00}\right]\right\}
\end{aligned}
$$


and the stresses and electric displacements

$$
\begin{aligned}
\mathbf{t}_{1}=\left(1-\frac{\left|x_{1}\right|}{\sqrt{x_{1}^{2}-1}}\right)\left(\mathbf{N}_{1}^{T} \mathbf{S}^{T}+\mathbf{N}_{3} \mathbf{H}\right) \phi^{00} & \\
+ & \varepsilon\left\{\frac { 3 } { 2 } \left(1-2 x_{1}^{2}+2\left|x_{1}\right| \sqrt{\left.x_{1}^{2}-1\right)}\left(\mathbf{N}_{3} \mathbf{S}-\mathbf{N}_{1}^{T} \mathbf{L}\right) \boldsymbol{\gamma}_{2}^{1, \infty}\right.\right. \\
& -\left(\mathbf{N}_{1}^{T} \mathbf{S}^{T} \mathbf{H}^{-1}+\mathbf{N}_{3}\right)\left[\left(1-\frac{\left|x_{1}\right|}{\sqrt{x_{1}^{2}-1}}\right)\left(\omega_{1} \mathbf{m}-\frac{5}{2} \mathbf{N}_{2} \phi^{00}+3 \mathbf{S} \boldsymbol{\gamma}_{2}^{1, \infty}\right)\right. \\
& \left.\left.+\frac{3}{2}\left(1+2 x_{1}^{2}-\frac{2\left|x_{1}\right|^{3}}{\sqrt{x_{1}^{2}-1}}\right)\left(\mathbf{N}_{2} \boldsymbol{\phi}^{00}-\mathbf{S} \boldsymbol{\gamma}_{2}^{1, \infty}\right)\right]\right\} \\
\mathbf{t}_{2}= & -\left(1-\frac{\left|x_{1}\right|}{\sqrt{x_{1}^{2}-1}}\right) \mathbf{S}^{T} \boldsymbol{\phi}^{00}+\varepsilon\left\{\frac{3}{2}\left(1-2 x_{1}^{2}+2\left|x_{1}\right| \sqrt{x_{1}^{2}-1}\right) \mathbf{L} \boldsymbol{\gamma}_{2}^{1, \infty}\right. \\
& +\mathbf{S}^{T} \mathbf{H}^{-1}\left[\left(1-\frac{\left|x_{1}\right|}{\sqrt{x_{1}^{2}-1}}\right)\left(\omega_{1} \mathbf{m}-\frac{5}{2} \mathbf{N}_{2} \phi^{00}+3 \mathbf{S} \boldsymbol{\gamma}_{2}^{1, \infty}\right)\right. \\
& \left.\left.+\frac{3}{2}\left(1+2 x_{1}^{2}-\frac{2\left|x_{1}\right|^{3}}{\sqrt{x_{1}^{2}-1}}\right)\left(\mathbf{N}_{2} \phi^{00}-\mathbf{S} \boldsymbol{\gamma}_{2}^{1, \infty}\right)\right]\right\}
\end{aligned}
$$

To obtain the expressions (133-138) we have used the fact that the matrices SH, LS, $\mathbf{H}^{-1} \mathbf{S}$, and $\mathbf{S L}^{-1}$ are antisymmetric and verify the identity

$$
\mathbf{H L}-\mathbf{S S}=\mathbf{I}
$$

as it was proved, for instance, in Lothe and Barnett (1976). Note that the terms of $O(1)$, corresponding to $\varepsilon \rightarrow 0$ in the above formulae, provide the solution for a rigid line inclusion of electric conductor.

\section{Cracks.}

6.1. Electrically impermeable cracks. When the inhomogeneity is a cavity (crack), one can suppose that on its boundary the tractions and normal electric displacements are vanishing (e.g., Pak 1990, Sosa 1991, Suo et al. 1992). Such a condition reduces to

$$
\phi^{\infty}+\phi=0
$$

on the boundary $\mathcal{C}$ of the exterior domain. In the same way as for the piezoelectric inclusions, we obtain the $O(1)$-equations

$$
\begin{aligned}
\mathbf{t}_{2}^{\infty} x_{1}+\widetilde{\mathbb{K}}_{0}\left(-\mathbf{L} \mathbf{u}^{0}\left(x_{1}\right)+\mathbf{S} \phi^{0}\left(x_{1}\right)\right) & =\mathbf{0} \\
\widehat{\mathbb{L}_{0}} \phi^{0}\left(x_{1}\right) & =\mathbf{0}
\end{aligned}
$$

for which we have the solutions:

$$
\mathbf{u}^{0}\left(x_{1}\right)=-\mathbf{L}^{-1} \mathbf{t}_{2}^{\infty} x_{1} ; \quad \phi^{0}\left(x_{1}\right)=\mathbf{0} .
$$


Similarly, (56-57), in which $\mathbf{u}^{* n}\left(x_{1}\right)=\boldsymbol{\phi}^{* n}\left(x_{1}\right)=\mathbf{0}, n=0,1$. They have the solution

$$
\begin{aligned}
& \phi^{1}\left(x_{1}\right)=\sqrt{1-x_{1}^{2}} \frac{d}{d x_{1}}\left\{\mathbf{t}_{1}^{\infty} \sqrt{D\left(x_{1}\right)}-\mathcal{N}_{1}^{u} \mathbf{u}^{0}\left(x_{1}\right)\right\} \\
& \mathbf{u}^{1}\left(x_{1}\right)=\mathbf{L}^{-1} \mathbf{S}^{T} \phi^{1}\left(x_{1}\right)-\mathbf{L}^{-1} \mathbb{C}^{-1}\left\{\mathbf{t}_{1}^{\infty} S\left(x_{1}\right)-\left(\mathcal{M}_{1}^{u} \mathbf{u}^{0}\left(x_{1}\right)\right)\right\}
\end{aligned}
$$

with $\mathbb{C}^{-1}$ being the solution operator of the Carleman equation, defined in (122). In (144-145) the operators $\mathcal{M}$ and $\mathcal{N}$ are given by (64) and (67).

The formulae (144-145) allow us to obtain the solution of $O(\varepsilon)$ as:

$$
\phi^{1}\left(x_{1}\right)=\sqrt{1-x_{1}^{2}} \frac{D^{\prime}\left(x_{1}\right)}{2 \sqrt{D\left(x_{1}\right)}} \mathbf{t}_{1}^{\infty} ; \quad \mathbf{u}^{1}\left(x_{1}\right)=\mathbf{L}^{-1} \mathbf{S}^{T} \phi^{1}\left(x_{1}\right)-\mathbf{L}^{-1} \mathbf{t}_{1}^{\infty} \mathbb{C}^{-1}\left\{S\left(x_{1}\right)\right\} .
$$

Consider a lemon-shaped cavity. The densities $\mathbf{u}^{0}$ and $\phi^{0}$ are those in (143) and from (146) we obtain

$$
\mathbf{u}^{1}\left(x_{1}\right)=-3 x_{1}\left(1-x_{1}^{2}\right) \mathbf{L}^{-1} \mathbf{S}^{T} \mathbf{t}_{1}^{\infty} ; \quad \phi^{1}\left(x_{1}\right)=-3 x_{1}\left(1-x_{1}^{2}\right) \mathbf{t}_{1}^{\infty} .
$$

The displacements and the electric potential on the axis $O x_{1}$, for $\left|x_{1}\right|>1$, take the form

$$
\begin{gathered}
\mathbf{u}\left(x_{1}\right)=-\left(\operatorname{sgn}\left(x_{1}\right) \sqrt{x_{1}^{2}-1}-x_{1}\right) \mathbf{S} \mathbf{L}^{-1} \mathbf{t}_{2}^{\infty}+\varepsilon\left\{\left[\frac{1}{4}\left(\operatorname{sgn}\left(x_{1}\right) \sqrt{x_{1}^{2}-1}-x_{1}\right)^{3}\right.\right. \\
\left.\left.-\frac{3}{4}\left(\operatorname{sgn}\left(x_{1}\right) \sqrt{x_{1}^{2}-1}-x_{1}\right)\right]\left(\mathbf{S}+\mathbf{H L}^{-1} \mathbf{S}^{T}\right) \mathbf{t}_{1}^{\infty}\right\},
\end{gathered}
$$

while the stresses and the electric displacements become

$$
\begin{aligned}
\mathbf{t}_{1}= & -\left(1-\frac{\left|x_{1}\right|}{\sqrt{x_{1}^{2}-1}}\right)\left(\mathbf{N}_{3} \mathbf{S L}^{-1}-\mathbf{N}_{1}^{T}\right) \mathbf{t}_{2}^{\infty} \\
& -\varepsilon \frac{3}{2}\left(1-2 x_{1}^{2}+2\left|x_{1}\right| \sqrt{x_{1}^{2}-1}\right) \mathbf{N}_{3} \mathbf{L}^{-1} \mathbf{t}_{1}^{\infty} \\
\mathbf{t}_{2} & =-\left(1-\frac{\left|x_{1}\right|}{\sqrt{x_{1}^{2}-1}}\right) \mathbf{t}_{2}^{\infty}+O\left(\varepsilon^{2}\right) .
\end{aligned}
$$

Remark that the terms of $O(1)$ in $(148-150)$ corresponding to a crack line $(\varepsilon \rightarrow 0)$, represent the solution for an electrically impermeable crack embedded in an infinite piezoelectric body. At $x_{1}=1$, we obtain the classical expressions of the intensity factors (see for instance Suo et al. 1992):

$$
\mathbf{K}=\lim _{x_{1} \rightarrow 1}\left(\sqrt{2 \pi\left(x_{1}-1\right)} \mathbf{t}_{2}\left(x_{1}\right)\right)=\sqrt{\pi} \mathbf{t}_{2}^{\infty}+O\left(\varepsilon^{2}\right),
$$

where $\mathbf{K}=\left(K_{I I}, K_{I}, K_{I I I}, K_{I V}\right)$. There are no contributions of $O(\varepsilon)$ in these coefficients since such terms are absent from (150).

6.2. Cracks with inside electric field. A more refined model for real flaws in electroelastic bodies is that in which an inside electric field is allowed in the cavity (e.g., Sosa and Khutoryansky 1996). In this case, the interface boundary conditions become

$$
\phi_{i}^{\infty}+\phi_{i}=\delta_{i 4} \phi_{4}^{*}, \quad u_{4}^{\infty}+u_{4}=u_{4}^{*}
$$

for $i=1,2,3,4$. If $\varepsilon_{0}$ is the dielectric permitivity in the inside domain (vacuum, air), such that $\mathbf{D}^{*}=\varepsilon_{0} \mathbf{E}^{*}$, then

$$
E_{i}^{*}=-\frac{\partial u_{4}^{*}}{\partial x_{i}}, \quad D_{1}^{*}=-\frac{\partial \phi_{4}^{*}}{\partial x_{2}}, \quad D_{2}^{*}=\frac{\partial \phi_{4}^{*}}{\partial x_{1}} .
$$


Introducing a single nonvanishing component $F_{4}^{*}=\varepsilon_{0} u_{4}^{*}+i \phi_{4}^{*}$ of $\mathbf{F}^{*}$ one can perform similar calculations as in Sec. 3 in order to obtain real systems for the densities (37.38). The system of $O(1)$ reads

$$
\begin{aligned}
-E_{1}^{\infty} x_{1}+\widetilde{\mathbb{K}}_{0}\left(S_{4 i} u_{i}^{0}\left(x_{1}\right)+H_{4 i} \phi_{i}^{0}\left(x_{1}\right)\right) & =u_{4}^{0 *}\left(x_{1}\right) \\
\widehat{\mathbb{L}}_{0} u_{4}^{0}\left(x_{1}\right) & =0 \\
t_{2 i}^{\infty} x_{1}+\widetilde{\mathbb{K}}_{0}\left(-L_{i j} u_{j}^{0}\left(x_{1}\right)+S_{j i} \phi_{j}^{0}\left(x_{1}\right)\right) & =\delta_{i 4} \phi_{4}^{0 *} \\
\widehat{\mathbb{L}}_{0} \phi_{i}^{0}\left(x_{1}\right) & =0
\end{aligned}
$$

for which we have the solutions:

$$
\begin{gathered}
\mathbf{u}^{0}\left(x_{1}\right)=\mathbf{U}^{0} x_{1}, \quad \phi^{0}\left(x_{1}\right)=\mathbf{0} \\
u_{4}^{0 *}\left(x_{1}\right)=U_{4}^{0 *} x_{1}, \quad \phi_{4}^{0 *}\left(x_{1}\right)=\Phi_{4}^{0 *} x_{1} \\
U_{i}^{0} \equiv-\bar{L}_{i j}^{-1} t_{2 j}^{\infty}, U_{4}^{0} \equiv 0, U_{4}^{0 *} \equiv-\left(E_{1}^{\infty}+S_{4 i} \bar{L}_{i j}^{-1} t_{2 j}^{\infty}\right), \Phi_{4}^{0 *} \equiv\left(D_{2}-L_{4 i} \bar{L}_{i j}^{-1} t_{2 j}^{\infty}\right)
\end{gathered}
$$

for $i, j=1,2,3$. Here $\bar{L}_{i j}^{-1}$ is the inverse of the $3 \times 3$ matrix $\left(L_{i, j}\right) i, j=1,2,3$. That such an inverse exists is proved in [1].

By taking into account the above solution, the system of $O(\varepsilon)$ becomes:

$$
\begin{gathered}
-E_{2}^{\infty} S\left(x_{1}\right)+\frac{1}{\pi} S_{4 i} \int_{-1}^{1} \frac{u_{i}^{1}(t)}{\sqrt{1-t^{2}}} \ln \left|x_{1}-t\right| d t \\
+\frac{1}{\pi} H_{4 i} \int_{-1}^{1} \frac{\phi_{i}^{1}(t)}{\sqrt{1-t^{2}}} \ln \left|x_{1}-t\right| d t+\left(\mathcal{K}_{1}^{u} \mathbf{u}^{0}\right)_{4}\left(x_{1}\right) \\
=u_{4}^{1 *}\left(x_{1}\right)+\frac{S\left(x_{1}\right)}{2 \varepsilon_{0}} U_{4}^{0 *} \\
-E_{2}^{\infty} \sqrt{D\left(x_{1}\right)}-\int_{x_{1}}^{1} \frac{u_{4}^{1}(t) d t}{\sqrt{1-t^{2}}}+\left(\mathcal{L}_{1}^{u} \mathbf{u}^{0}\right)_{4}\left(x_{1}\right)=\frac{\sqrt{D\left(x_{1}\right)}}{2 \varepsilon_{0}} U_{4}^{0 *} \\
-t_{1 i}^{\infty} S\left(x_{1}\right)-\frac{1}{\pi} L_{i j} \int_{-1}^{1} \frac{u_{j}^{1}(t)}{\sqrt{1-t^{2}}} \ln \left|x_{1}-t\right| d t \\
+\frac{1}{\pi} S_{j i} \int_{-1}^{1} \frac{\phi_{j}^{1}(t)}{\sqrt{1-t^{2}}} \ln \left|x_{1}-t\right| d t+\left(\mathcal{M}_{1}^{u} \mathbf{u}^{0}\right)_{i}\left(x_{1}\right) \\
=\delta_{i 4}\left[\phi_{4}^{1 *}\left(x_{1}\right)+\frac{S\left(x_{1}\right)}{2 \varepsilon_{0}} \Phi_{4}^{0 *}\right] \\
-t_{1 i}^{\infty} \sqrt{D\left(x_{1}\right)}-\int_{x_{1}}^{1} \frac{\phi_{i}^{1}(t) d t}{\sqrt{1-t^{2}}}+\left(\mathcal{N}_{1}^{u} \mathbf{u}^{0}\right)_{i}\left(x_{1}\right)=\delta_{i 4}\left(\frac{\sqrt{D\left(x_{1}\right)}}{2 \varepsilon_{0}} \Phi_{4}^{0 *}\right)
\end{gathered}
$$

where the operators are those defined in (58-69). 
The system (161-164) has the following solution:

$$
\begin{aligned}
u_{i}^{1}\left(x_{1}\right) & =-\bar{L}_{i j}^{-1} L_{j 4} u_{4}^{1}+\bar{L}_{i j}^{-1} \mathbb{C}^{-1}\left\{-t_{1 j}^{\infty} S\left(x_{1}\right)+\left(\mathbf{S}^{T} \mathbf{N}_{3} \mathbf{U}^{0}\right)_{j} \frac{1}{\pi} \int_{-1}^{1} \frac{S(t) t d t}{\left(t-x_{1}\right) \sqrt{1-t^{2}}}\right. \\
& \left.-\left(\mathbf{S}^{T} \mathbf{V}\right)_{j} \frac{1}{\pi} \int_{-1}^{1} \frac{\sqrt{D(t)} d t}{t-x_{1}}+\frac{x_{1} \sqrt{D\left(x_{1}\right)}}{\sqrt{1-x_{1}^{2}}}\left(\mathbf{N}_{3} \mathbf{U}^{0}\right)_{j}-S\left(x_{1}\right)\left(\left(\mathbf{N}_{3} \mathbf{S}-\mathbf{N}_{1}^{T} \mathbf{L}\right) \mathbf{U}^{0}\right)_{j}\right\}
\end{aligned}
$$

$$
\begin{aligned}
u_{4}^{1}\left(x_{1}\right)=- & \left(\mathbf{N}_{1} \mathbf{U}^{0}\right)_{4}\left[x_{1} S^{\prime}\left(x_{1}\right)+\frac{S\left(x_{1}\right)}{1-x_{1}^{2}}\right] \\
& +\sqrt{1-x_{1}^{2}} \frac{D^{\prime}\left(x_{1}\right)}{2 \sqrt{D\left(x_{1}\right)}} W \\
\phi_{k}^{1}\left(x_{1}\right)=- & {\left[x_{1} S^{\prime}\left(x_{1}\right)+\frac{S\left(x_{1}\right)}{1-x_{1}^{2}}\right]\left(\mathbf{N}_{3} \mathbf{U}^{0}\right)_{k} } \\
& +\sqrt{1-x_{1}^{2}} \frac{D^{\prime}\left(x_{1}\right)}{2 \sqrt{D\left(x_{1}\right)}} V_{k}
\end{aligned}
$$

$$
\begin{aligned}
u_{4}^{1 *}\left(x_{1}\right)= & -S\left(x_{1}\right)\left(\frac{U_{4}^{0 *}}{2 \varepsilon_{0}}+E_{2}^{\infty}\right) \\
& +\frac{1}{\pi} S_{4 k} \int_{-1}^{1} \frac{u_{k}^{1}(t)}{\sqrt{1-t^{2}}} \ln \left|x_{1}-t\right| d t+\frac{1}{\pi} H_{4 k} \int_{-1}^{1} \frac{\phi_{k}^{1}(t)}{\sqrt{1-t^{2}}} \ln \left|x_{1}-t\right| d t \\
& +\frac{x_{1} \sqrt{D\left(x_{1}\right)}}{\sqrt{1-x_{1}^{2}}}\left(\mathbf{N}_{1} \mathbf{U}^{0}\right)_{4}-S\left(x_{1}\right)\left[\left(\mathbf{N}_{1} \mathbf{S}-\mathbf{N}_{2} \mathbf{L}\right) \mathbf{U}^{0}\right]_{4}
\end{aligned}
$$

$$
\begin{aligned}
\phi_{4}^{1 *}\left(x_{1}\right)= & -S\left(x_{1}\right)\left(\frac{\Phi_{4}^{0 *}}{2 \varepsilon_{0}}+t_{14}^{\infty}\right) \\
& -\frac{1}{\pi} L_{4 k} \int_{-1}^{1} \frac{u_{k}^{1}(t)}{\sqrt{1-t^{2}}} \ln \left|x_{1}-t\right| d t+\frac{1}{\pi} S_{k 4} \int_{-1}^{1} \frac{\phi_{k}^{1}(t)}{\sqrt{1-t^{2}}} \ln \left|x_{1}-t\right| d t \\
& +\frac{x_{1} \sqrt{D\left(x_{1}\right)}}{\sqrt{1-x_{1}^{2}}}\left(\mathbf{N}_{3} \mathbf{U}^{0}\right)_{4}-\left[\left(\mathbf{N}_{3} \mathbf{S}-\mathbf{N}_{1}^{T} \mathbf{L}\right) \mathbf{U}^{0}\right]_{4}
\end{aligned}
$$

with $i, j=1,2,3$ and $k=1,2,3,4$. We have used the notations

$$
\begin{aligned}
& V_{k}=t_{1 k}^{\infty}+\delta_{k 4} \frac{1}{2 \varepsilon_{0}} \Phi_{4}^{0 *}+\left(\left(\mathbf{N}_{3} \mathbf{S}-\mathbf{N}_{1}^{T} \mathbf{L}\right) \mathbf{U}^{0}\right)_{k} \\
& W=\left(\left(\mathbf{N}_{1} \mathbf{S}-\mathbf{N}_{2} \mathbf{L}\right) \mathbf{U}^{0}\right)_{4}+\frac{U_{4}^{0 *}}{2 \varepsilon_{0}}+E_{2}^{\infty} .
\end{aligned}
$$

Let us determine the real expressions of the generalized displacements and stresses for a lemon-shaped cavity. 
The displacements and the electric potential on the axis $O x_{1}$, for $\left|x_{1}\right|>1$, take the form

$$
\begin{gathered}
u_{i}\left(x_{1}\right)=\left(\operatorname{sgn}\left(x_{1}\right) \sqrt{x_{1}^{2}-1}-x_{1}\right) S_{i j} U_{j}^{0} \\
+\frac{\varepsilon}{4}\left\{\left[2\left(S_{i j} \bar{L}_{j k}^{-1}\left(\mathbf{N}_{3} \mathbf{U}^{0}\right)_{k}\right)+3\left((\mathbf{S X})_{i}-(\mathbf{H V})_{i}\right)\right]\left(\operatorname{sgn}\left(x_{1}\right) \sqrt{x_{1}^{2}-1}-x_{1}\right)\right. \\
\left.-\left((\mathbf{S X})_{i}-(\mathbf{H V})_{i}\right)\left(\operatorname{sgn}\left(x_{1}\right) \sqrt{x_{1}^{2}-1}-x_{1}\right)^{3}\right\}
\end{gathered}
$$

with

$$
X_{j}=-\bar{L}_{j k}^{-1}\left[\left(\mathbf{S}^{T} \mathbf{V}\right)_{k}-L_{k 4} W+\left(\mathbf{N}_{3} \mathbf{U}^{0}\right)_{k}\right], \quad X_{4}=-W .
$$

In (172-174) we have $i=1,2,3,4$ while $j, k=1,2,3$.

In order to obtain the stresses and the electric displacements, we use (86-87). For a lemon-shaped geometry, we have $\tilde{c}_{2}=\tilde{d}_{2}=0$ in (203) and from (205) we deduce that $\mathbb{H}=\mathbb{R}_{1}$ and it results that

$$
\begin{aligned}
t_{1 i}= & \left(1-\frac{\left|x_{1}\right|}{\sqrt{x_{1}^{2}-1}}\right)\left(\left(\mathbf{N}_{3} \mathbf{S}-\mathbf{N}_{1}^{T} \mathbf{L}\right) \mathbf{U}^{0}\right)_{i} \\
& +\varepsilon\left\{\frac{1}{2}\left(1-\frac{\left|x_{1}\right|}{\sqrt{x_{1}^{2}-1}}\right)\left(\mathbf{N}_{3} \mathbf{S}-\mathbf{N}_{1}^{T} \mathbf{L}\right)_{i j} \bar{L}_{j k}^{-1}\left(\mathbf{N}_{3} \mathbf{U}^{0}\right)_{k}\right. \\
& \left.+\frac{3}{2}\left(1-2 x_{1}^{2}+2\left|x_{1}\right| \sqrt{x_{1}^{2}-1}\right)\left[\left(\mathbf{N}_{3} \mathbf{S}-\mathbf{N}_{1}^{T} \mathbf{L}\right) \mathbf{X}-\left(\mathbf{N}_{1}^{T} \mathbf{S}^{T}+\mathbf{N}_{3} \mathbf{H}\right) \mathbf{V}\right]_{i}\right\} \\
& t_{2 i}=\left(1-\frac{\left|x_{1}\right|}{\sqrt{x_{1}^{2}-1}}\right)\left(\mathbf{L} \mathbf{U}^{0}\right)_{i}+\varepsilon\left\{\frac{1}{2}\left(1-\frac{\left|x_{1}\right|}{\sqrt{x_{1}^{2}-1}}\right) L_{i j} \bar{L}_{j k}^{-1}\left(\mathbf{N}_{3} \mathbf{U}^{0}\right)_{k}\right. \\
& \left.\quad+\frac{3}{2}\left(1-2 x_{1}^{2}+2\left|x_{1}\right| \sqrt{x_{1}^{2}-1}\right)\left(\mathbf{L X}+\mathbf{S}^{T} \mathbf{V}\right)_{i}\right\}
\end{aligned}
$$

for $i=1,2,3,4$ while $j, k=1,2,3$. At $x_{1}=1$, the stress intensity factors result as

$$
\begin{aligned}
& K_{j}=\sqrt{\pi}\left(\sigma_{2 j}^{\infty}+\frac{\varepsilon}{2}\left(\mathbf{N}_{3}\right)_{j k} \bar{L}_{k l}^{-1} \sigma_{2 l}^{\infty}\right) \\
& K_{4}=\sqrt{\pi}\left(L_{4 j} \bar{L}_{j k}^{-1} \sigma_{2 k}^{\infty}+\frac{\varepsilon}{2} L_{4 j} \bar{L}_{j k}^{-1}\left(\mathbf{N}_{3}\right)_{k l} \bar{L}_{l m}^{-1} \sigma_{2 m}^{\infty}\right)=L_{4 j} \bar{L}_{j k}^{-1} K_{k} .
\end{aligned}
$$

The last relation shows that the electric displacement intensity factor $K_{4}$ is not independent from the corresponding coefficients of the stresses. This shows that the electric fields are not singular at the endpoints of the cavity.

Remark that in this case the terms of $O(\varepsilon)$ in (178) represent an influence of the anisotropy on the stress intensity factors. Such an influence was experimentally observed (e.g., Pisarenko et al. 1985). This property is a consequence of the present model involving inside electric fields, since in (151) such an influence is missing.

7. Conclusions. Uniform valid asymptotic solutions for lamellar inhomogeneities embedded in infinite piezoelectric materials were obtained. We have studied the case of piezoelectric inclusions, rigid inclusions of electric conductor, electrically impermeable cavities and cavities with inside electric field. The solutions have been obtained 
for arbitrary-shaped lamellar inhomogeneities. The first two terms in the asymptotic expansion of the solution were given. Real-form expressions of the elastic and electric fields at the common boundary of the two bodies (or on the hole boundary) and on the Ox $x_{1}$-axis, outside the lamellar domain, have been obtained.

As concrete examples, we considered the case of an elliptic lamellar piezoelectric inclusion, corresponding to finite curvature radius at the ends, and also the case of a lemon-shaped inclusion, when we have a zero radius of curvature at the ends of the body. It was found that, while for elliptic piezoelectric inclusions the perturbation stresses and clectric displacements at the inclusion ends have the same order as the fields given at infinity, for a lemon-shaped inclusion they are an order-of-magnitude smaller. The result is important since the stress/electric displacement concentrations may strongly affect the mechanical resistance of composite structures.

We have also considered holes with electrically impermeable boundaries and holes with inside clectric ficlds. The first two terms in the asymptotic expansions of the intensity factors were calculated for lemon-shaped cavities. It was deduced that, when inside electric fields are considered, these stress intensity coefficients are influenced by the anisotropy of the piezoelectric material. Such an influence was experimentally observed, but not predicted, by classical crack models. It appears that the finite-thickness cavities with cusps are more realistic than the classical line models for cracks in piezoelectric bodies. Further investigations are necessary in order to compare the theoretical predictions with the experimental results.

A. Asymptotic expansion of integral operators. In this Appendix we shall show how the method of Handelsman and Keller (1967) can be modified to obtain the asymptotic expansion as $\varepsilon \rightarrow 0$ of the integral operators appearing in the previous sections.

We first consider the integral operator defined by the relation (31). By an obvious change of variables we can write

$$
\begin{aligned}
& F_{\alpha}\left(x_{1}+p_{\alpha} \varepsilon Y_{ \pm}\left(x_{1}\right)\right) \\
&=-\frac{1}{\pi i} \int_{c_{\alpha}}^{d_{\alpha}} \frac{\tilde{f}_{\alpha}(\xi)}{\sqrt{\left(d_{\alpha}-\xi\right)\left(\xi-c_{\alpha}\right)}} \ln \left(x_{1}+p_{\alpha} \varepsilon Y_{ \pm}\left(x_{1}\right)-\xi\right) d \xi \\
&=-\frac{1}{\pi i} \ln \left(\frac{d_{\alpha}-c_{\alpha}}{2}\right) \int_{-1}^{1} \frac{f_{\alpha}(t)}{\sqrt{1-t^{2}}} d t \\
&-\frac{1}{\pi i} \int_{-1}^{1} \frac{f_{\alpha}(t)}{\sqrt{1-t^{2}}} \ln \left(x_{1}-t+p_{\alpha} \varepsilon Y_{ \pm}\left(x_{1}\right)+\varepsilon^{2} l_{\alpha}\left(x_{1}\right)+O\left(\varepsilon^{3}\right)\right) d t .
\end{aligned}
$$

Here we shall assume that $c_{\alpha}$ and $d_{\alpha}$ have asymptotic developments of the form

$$
c_{\alpha}(\varepsilon)=-1+c_{\alpha 2} \varepsilon^{2}+O\left(\varepsilon^{3}\right) ; \quad d_{\alpha}(\varepsilon)=1-d_{\alpha 2} \varepsilon^{2}+O\left(\varepsilon^{3}\right),
$$

where the constants $c_{\alpha 2}$ and $d_{\alpha 2}$ will be determined shortly. It can be shown, similarly as in Homentscovschi (1982), that the terms of $O(\varepsilon)$ in $c_{\alpha}(\varepsilon)$ and $d_{\alpha}(\varepsilon)$ must vanish. We have also denoted

$$
l_{\alpha}\left(x_{1}\right)=\frac{c_{\alpha 2}+d_{\alpha 2}}{2} x_{1}+\frac{d_{\alpha 2}-c_{\alpha 2}}{2} .
$$


The analysis in Sec. 8 of Homentcovschi (1982), where we replace $z$ by $z_{\alpha}$, yields the asymptotic formulae

$$
\begin{aligned}
& \frac{1}{2}\left(F_{\alpha}\left(z_{\alpha+}\right)+F_{\alpha}\left(z_{\alpha-}\right)\right)=-\frac{1}{\pi i} \int_{-1}^{1} \frac{f_{\alpha}(t)}{\sqrt{1-t^{2}}} \ln \left|x_{1}-t\right| d t \\
& +\varepsilon p_{\alpha}\left[\frac{\sqrt{D\left(x_{1}\right)}}{\sqrt{1-x_{1}^{2}}} f_{\alpha}\left(x_{1}\right)-i S\left(x_{1}\right)\left(\mathbb{R}_{1} f_{\alpha}\right)\left(x_{1}\right)\right] \\
& +\varepsilon^{2}\left\{\frac{c_{\alpha 2}+d_{\alpha 2}}{2 i}\left(\mathbb{R}_{0} f_{\alpha}\right)\left(x_{1}\right)-i l_{\alpha}\left(x_{1}\right)\left(\mathbb{R}_{1} f_{\alpha}\right)\left(x_{1}\right)+\frac{p_{\alpha}^{2} x_{1}}{\sqrt{1-x_{1}^{2}}} \frac{S\left(x_{1}\right) \sqrt{D\left(x_{1}\right)}}{1-x_{1}^{2}} f_{\alpha}\left(x_{1}\right)\right. \\
& \left.+\frac{p_{\alpha}^{2} S\left(x_{1}\right) \sqrt{D\left(x_{1}\right)}}{\sqrt{1-x_{1}^{2}}} f_{\alpha}^{(1)}\left(x_{1}\right)-i \frac{p_{\alpha}^{2}\left(S^{2}\left(x_{1}\right)+D\left(x_{1}\right)\right)}{2}\left(\mathbb{R}_{2} f_{\alpha}\right)\left(x_{1}\right)\right\}+O\left(\varepsilon^{3}\right) \\
& \frac{1}{2}\left(F_{\alpha}\left(z_{\alpha+}\right)-F_{\alpha}\left(z_{\alpha-}\right)\right) \\
& =-\int_{x_{1}}^{1} \frac{f_{\alpha}(t)}{\sqrt{1-t^{2}}} d t+\varepsilon p_{\alpha}\left[\frac{S\left(x_{1}\right)}{\sqrt{1-x_{1}^{2}}} f_{\alpha}\left(x_{1}\right)-i \sqrt{D\left(x_{1}\right)}\left(\mathbb{R}_{1} f_{\alpha}\right)\left(x_{1}\right)\right] \\
& +\varepsilon^{2}\left\{\left[l_{\alpha}\left(x_{1}\right)+\frac{p_{\alpha}^{2} x_{1}}{2\left(1-x_{1}^{2}\right)}\left(S^{2}\left(x_{1}\right)+D\left(x_{1}\right)\right)\right] \frac{f_{\alpha}\left(x_{1}\right)}{\sqrt{1-x_{1}^{2}}}\right. \\
& +\frac{p_{\alpha}^{2}}{\left.2 \sqrt{1-x_{1}^{2}}\left(S^{2}\left(x_{1}\right)+D\left(x_{1}\right)\right) f_{\alpha}^{(1)}\left(x_{1}\right)-i p_{\alpha}^{2} S\left(x_{1}\right) \sqrt{D\left(x_{1}\right)}\left(\mathbb{R}_{2} f_{\alpha}\right)\left(x_{1}\right)\right\}+O\left(\varepsilon^{3}\right) .}
\end{aligned}
$$

We have denoted

$$
\left(\mathbb{R}_{n} f_{\alpha}\right)\left(x_{1}\right)=\frac{1}{\pi} \int_{-1}^{1} \frac{f_{\alpha}(t)}{\sqrt{1-t^{2}}} \frac{d t}{\left(t-x_{1}\right)^{n}} .
$$

In the case $\left|x_{1}\right|<1$ and $n \geq 1$ the integral will be interpreted in the Hadamard sense, by retaining the finite part only.

All the terms in the expansions (183-184) except

$$
\left[l_{\alpha}\left(x_{1}\right)+\frac{p_{\alpha}^{2} x_{1}}{2\left(1-x_{1}^{2}\right)}\left(S^{2}\left(x_{1}\right)+D\left(x_{1}\right)\right)\right] \frac{f_{\alpha}\left(x_{1}\right)}{\sqrt{1-x_{1}^{2}}}
$$

are regular for $x_{1} \in[-1,1]$. In order that the term (186) be also regular we have to impose the constraints

$$
l_{\alpha}(1)-\frac{p_{\alpha}^{2} D^{(1)}(1)}{4}=0 ; \quad l_{\alpha}(-1)-\frac{p_{\alpha}^{2} D^{(1)}(-1)}{4}=0
$$

which yield

$$
c_{\alpha 2}=-\frac{p_{\alpha}^{2} D^{(1)}(-1)}{4} ; \quad d_{\alpha 2}=\frac{p_{\alpha}^{2} D^{(1)}(1)}{4} .
$$

Hence, we can write

$$
c_{\alpha 2}=-p_{\alpha}^{2} \tilde{c}_{2} ; \quad d_{\alpha 2}=-p_{\alpha}^{2} \tilde{d}_{2} ; \quad l_{\alpha}\left(x_{1}\right)=-p_{\alpha}^{2} \tilde{l}\left(x_{1}\right),
$$


where

$$
\tilde{c}_{2}=\frac{D^{(1)}(-1)}{4} ; \quad \tilde{d}_{2}=-\frac{D^{(1)}(1)}{4} ; \quad \tilde{l}\left(x_{1}\right)=\frac{\tilde{d}_{2}+\tilde{c}_{2}}{2} x_{1}+\frac{\tilde{d}_{2}-\tilde{c}_{2}}{2}
$$

depend only upon the geometry of the domain.

Finally, the relations (183-184) yield the asymptotic formulae (32-33) where the operators $\mathbb{K}_{m}$ and $\mathbb{L}_{m}$ are given by

$$
\begin{gathered}
\left(\mathbb{K}_{0} f_{\alpha}\right)\left(x_{1}\right)=-\frac{1}{\pi i} \int_{-1}^{1} \frac{f_{\alpha}(t)}{\sqrt{1-t^{2}}} \ln \left|x_{1}-t\right| d t \\
\left(\mathbb{K}_{1} f_{\alpha}\right)\left(x_{1}\right)=\frac{\sqrt{D\left(x_{1}\right)}}{\sqrt{1-x_{1}^{2}}} f_{\alpha}\left(x_{1}\right)-i S\left(x_{1}\right)\left(\mathbb{R}_{1} f_{\alpha}\right)\left(x_{1}\right) \\
\left(\mathbb{L}_{0} f_{\alpha}\right)\left(x_{1}\right)=-\int_{x_{1}}^{1} \frac{f_{\alpha}(t)}{\sqrt{1-t^{2}}} d t \\
\left(\mathbb{L}_{1} f_{\alpha}\right)\left(x_{1}\right)=\frac{S\left(x_{1}\right)}{\sqrt{1-x_{1}^{2}}} f_{\alpha}\left(x_{1}\right)-i \sqrt{D\left(x_{1}\right)}\left(\mathbb{R}_{1} f_{\alpha}\right)\left(x_{1}\right) .
\end{gathered}
$$

We can also write

$$
F_{\alpha}\left(z_{\alpha \pm}\right)=\sum_{m=0}^{1}\left(\varepsilon p_{\alpha}\right)^{m}\left(\left(\mathbb{K}_{m} \pm \mathbb{L}_{m}\right) f_{\alpha}\right)\left(x_{1}\right)+O\left(\varepsilon^{2}\right) .
$$

For $\left|x_{1}\right|>1$, the asymptotic expansion of the function $F_{\alpha}\left(x_{1}\right)$ can be directly obtained as

$$
\begin{aligned}
F_{\alpha}\left(x_{1}\right) & =-\frac{1}{\pi i} \int_{-1}^{1} \frac{f_{\alpha}(t)}{\sqrt{1-t^{2}}} \ln \left(x_{1}-\frac{d_{\alpha}+c_{\alpha}}{2}+\frac{d_{\alpha}-c_{\alpha}}{2} t\right) d t \\
& =-\frac{1}{\pi i} \int_{-1}^{1} \frac{f_{\alpha}(t) d t}{\sqrt{1-t^{2}}} \ln \left(\frac{d_{\alpha}-c_{\alpha}}{2}\right)-\frac{1}{\pi i} \int_{-1}^{1} \frac{f_{\alpha}(t)}{\sqrt{1-t^{2}}} \ln \left(x_{1}-t+O\left(\varepsilon^{2}\right)\right) d t \\
& =i\left(\mathbb{R} f_{\alpha}\right)\left(x_{1}\right)+O\left(\varepsilon^{2}\right)
\end{aligned}
$$

where

$$
\left(\mathbb{R} f_{\alpha}\right)\left(x_{1}\right)=\frac{1}{\pi} \int_{-1}^{1} \frac{f_{\alpha}(t)}{\sqrt{1-t^{2}}} \ln \left(x_{1}-t\right) d t .
$$

We focus now on the derivative of the function $F_{\alpha}$ at points on the interface boundary. We have

$$
\frac{1}{\pi i} \int_{c_{\alpha}}^{d_{\alpha}} \frac{\tilde{f}_{\alpha}(\xi)}{\sqrt{\left(d_{\alpha}-\xi\right)\left(\xi-c_{\alpha}\right)}} \frac{d \xi}{\xi-z_{\alpha \pm}}=\frac{1}{\pi i} \int_{-1}^{1} \frac{f_{\alpha}(t)}{\sqrt{1-t^{2}}} \frac{d t}{t-\mathcal{Z}_{\alpha \pm}}
$$

where

$$
\mathcal{Z}_{\alpha \pm}=\frac{2}{d_{\alpha}-c_{\alpha}} z_{\alpha \pm}-\frac{d_{\alpha}+c_{\alpha}}{d_{\alpha}-c_{\alpha}}=x_{1}+\left(\varepsilon p_{\alpha}\right) Y_{ \pm}\left(x_{1}\right)-\left(\varepsilon p_{\alpha}\right)^{2} \tilde{l}\left(x_{1}\right)+O\left(\varepsilon^{3}\right) .
$$

To determine the asymptotic expansion, as $\varepsilon \rightarrow 0$, of (198) we apply the method used in Homentcovschi (1979), formula A.7, where we put $\alpha=-1, \beta=1$. In this way, we obtain the formula $(78)$ in which

$$
\begin{gathered}
\left(\mathbb{M}_{0}^{ \pm} f_{\alpha}\right)\left(x_{1}\right)=-i\left(\mathbb{R}_{1} f_{\alpha}\right)\left(x_{1}\right)+f_{\alpha}\left(x_{1}\right) h^{ \pm}\left(x_{1}\right) \\
\left(\mathbb{M}_{1}^{ \pm} f_{\alpha}\right)\left(x_{1}\right)=Y_{ \pm}\left(x_{1}\right)\left[-i\left(\mathbb{R}_{2} f_{\alpha}\right)\left(x_{1}\right)+f_{\alpha}^{(1)}\left(x_{1}\right) h^{ \pm}\left(x_{1}\right)\right]
\end{gathered}
$$


where the complex-valued function $h^{ \pm}\left(x_{1}\right)$ is

$$
h^{ \pm}\left(x_{1}\right)=\frac{1}{\sqrt{\left[1+x_{1}+\left(\varepsilon p_{\alpha}\right) Y_{ \pm}\left(x_{1}\right)-\left(\varepsilon p_{\alpha}\right)^{2} \tilde{l}\left(x_{1}\right)\right]\left[1-x_{1}-\left(\varepsilon p_{\alpha}\right) Y_{ \pm}\left(x_{1}\right)+\left(\varepsilon p_{\alpha}\right)^{2} \tilde{l}\left(x_{1}\right)\right]}} .
$$

Note that $h^{ \pm}\left(x_{1}\right)$ can be decomposed into real and imaginary parts by using the moduli and arguments of the complex quantities involved in the denominator.

We also need the asymptotic development of the derivative of $F_{\alpha}\left(z_{\alpha}\right)$, for $z_{\alpha}=x_{1}$ with $\left|x_{1}\right|>1$. This can be obtained straightforwardly in the form

$$
\begin{gathered}
\frac{1}{\pi i} \int_{c_{\alpha}}^{d_{\alpha}} \frac{\tilde{f}_{\alpha}(\xi)}{\sqrt{\left(d_{\alpha}-\xi\right)\left(\xi-c_{\alpha}\right)}} \frac{d \xi}{\xi-x_{1}} \\
=\left(1-\left(\varepsilon p_{\alpha}\right)^{2} \frac{\tilde{c}_{2}+\tilde{d}_{2}}{2}\right) \frac{1}{\pi i} \int_{-1}^{1} \frac{f_{\alpha}(t)}{\sqrt{1-t^{2}}} \frac{d t}{t-x_{1}+\left(\varepsilon p_{\alpha}\right)^{2} \tilde{l}\left(x_{1}\right)} \\
=\frac{f_{\alpha}\left(x_{1}\right)}{\pi i} \int_{-1}^{1} \frac{1}{\sqrt{1-t^{2}}} \frac{d t}{t-x_{1}+\left(\varepsilon p_{\alpha}\right)^{2} \tilde{l}\left(x_{1}\right)}+\frac{1}{\pi i} \int_{-1}^{1} \frac{f_{\alpha}(t)-f_{\alpha}\left(x_{1}\right)}{\sqrt{1-t^{2}}} \frac{d t}{t-x_{1}} \\
-\frac{\left(\varepsilon p_{\alpha}\right)^{2} \tilde{l}\left(x_{1}\right)}{\pi i} \int_{-1}^{1} \frac{1}{\sqrt{1-t^{2}} \frac{\left[f_{\alpha}(t)-f_{\alpha}\left(x_{1}\right)-\left(t-x_{1}\right) f_{\alpha}^{\prime}\left(x_{1}\right)\right] d t}{\left(t-x_{1}\right)\left(t-x_{1}+\left(\varepsilon p_{\alpha}\right)^{2} \tilde{l}\left(x_{1}\right)\right)}} \\
=-i\left(\mathbb{R}_{1} f_{\alpha}\right)\left(x_{1}\right)-i f_{\alpha}\left(x_{1}\right)\left[\frac{\operatorname{sgn}\left(x_{1}\right)}{\sqrt{x_{1}^{2}-1}}-g\left(x_{1}\right) f_{\alpha}^{\prime}\left(x_{1}\right)\right. \\
+\frac{\left(\varepsilon p_{\alpha}\right)^{2} \tilde{l}\left(x_{1}\right) f_{\alpha}^{\prime}\left(x_{1}\right)}{\left.i \sqrt{\left(x_{1}-\left(\varepsilon p_{\alpha}\right)^{2} \tilde{l}\left(x_{1}\right)\right)^{2}-1}+\left(\varepsilon p_{\alpha}\right)^{2}\left(x_{1}\right)\right)^{2}-1}
\end{gathered}
$$

Note that the last term represents a contribution of $O(\varepsilon)$ only at the endpoints $x_{1}= \pm 1$.

We introduced

$$
g\left(x_{1}\right)=\frac{1}{\sqrt{\left[x_{1}+1+\left(\varepsilon p_{\alpha}\right)^{2} \tilde{c}_{2}\right]\left[x_{1}-1-\left(\varepsilon p_{\alpha}\right)^{2} \tilde{d}_{2}\right]}} .
$$

For the derivative of $\mathbf{F}$ we get

$$
\frac{d \mathbf{F}}{d \mathbf{Z}}=-\mathbb{H}\left(i \mathbf{f}^{0}\right)-\varepsilon \mathbb{H}\left(i \mathbf{f}^{1}\right),
$$

with $\mathbb{H}$ given by

$$
\left(\mathbb{H} f_{\alpha}\right)\left(x_{1}\right)=\left(\mathbb{R}_{1} f_{\alpha}\right)\left(x_{1}\right)+f_{\alpha}\left(x_{1}\right)\left[\frac{\operatorname{sgn}\left(x_{1}\right)}{\sqrt{x_{1}^{2}-1}}-g\left(x_{1}\right)\right]+\frac{\left(\varepsilon p_{\alpha}\right)^{2} \tilde{l}\left(x_{1}\right) f_{\alpha}^{\prime}\left(x_{1}\right)}{i \sqrt{\left(x_{1}-\left(\varepsilon p_{\alpha}\right)^{2} \tilde{l}\left(x_{1}\right)\right)^{2}-1}},
$$

and where $\mathbb{R}_{1}$ is defined in (185).

\section{REFERENCES}

[1] Barnett, D. M. and Lothe, J., 1975, Dislocations and line charges in anisotropic piezoelectric insulators, Phys. Status Solidi B 67, 105-111 
[2] Chung, M. Y. and Ting, T. C. T., 1996, Piezoelectric solid with an elliptic inclusion or hole, Int. J. Solids Structures 33 (23), 3343-3361

[3] Dascalu, C., 1997, Electroelasticity equations and energy approaches to fracture, Int. J. Engng. Sci. 35, 1185-1196

[4] Dascalu, C. and Maugin, G. A.. 1995, On the dynamic fracture of piezoelectric materials, Q. J. Mech. Appl. Mat. 48, 237-251

[5] Dascalu, C. and Homentcovschi, D., 1999, Uniform asymptotic solutions for lamellar inhomogeneities in anisotropic elastic solids, SIAM J. Appl. Math. 60, 18-42

[6] Geer, J. F. and Keller, J. B., 1968, Uniform asymptotic solutions for potential flow around a thin airfoil and the electrostatic potential about a thin conductor, SIAM J. Appl. Math. 16, 75-101

[7] Homentcovschi, D., 1979, Conformal mapping of the domain exterior to a thin region. SIAM J. Math. Anal. 10, 1246-1257

[8] Homentcovschi, D., 1982, Uniform asymptotic solutions for the two-dimensional potential field problem with joining relations on the surface of a slender body, Int. J. Engng. Sci. 20, 753-767

[9] Homentcovschi, D., 1984, Uniform asymptotic solutions of two-dimensional problems of elasticity for the domain exterior to a thin region, SIAM J. Appl. Math. 44, 1-10

[10] Homentcovschi, D. and Dascalu, C., 2000, Uniform asymptotic solutions for lamellar inhomogeneities in plane elasticity. J. Mech. Phys. Solids 48, 153-173

[11] Liang, J., Han, J., Wang, B., and Du, S., 1995, Electroelastic modelling of anisotropic piezoelectric materials with an elliptic inclusion, Int. J. Solids Structures 32 (20), 2989-3000

[12] Lothe, J. and Barnett, D. M., 1976, Integral formalism for surface waves in piezoelectric crystals. Existence considerations, J. Appl. Phys. 47, 1799--1807

[13] Lu, P., Tan, M. J., and Liew, K. M., 2000, A further investigation of Green functions for a piezoelectric material with a cavity or a crack, Int. J. Solids Structures 37, 1065-1078

[14] Maugin, G. A., 1988, Continuum mechanics of electromagnetic solids, North-Holland, Amsterdam

[15] Pak, Y. E., 1990, Crack extension force in a piezoelectric material, J. Appl. Mech. 57, 647-653

[16] Pak, Y. E., 1992, Linear electroelastic fracture mechanics of piezoelectric materials, Int. J. Fracture 54, 79-100

[17] Park, S. B. and Sun C. T., 1995, Effect of electric field on fracture of piezoelectric ceramics, Int. J. Fracture 70, 203-216

[18] Pisarenko, G. G., Chushko, V. M., and Kovalev, S. P., 1985, Anisotropy of fracture toughness of piezoelectric ceramics, J. Am. Ceram. Soc. 68 (5), 259-265

[19] Sosa, H., 1991, Plane problems in piezoelectric media with defects, Int. J. Solids Structures 28, 491-505

[20] Sosa, H. and Khutoryansky, N., 1996, New developments concerning piezoelectric materials with defects, Int. J. Solids Structures 33, 3399-3414

[21] Suo, Z., Kuo, C.-M., Barnett, D. M., and Willis, J. R., 1992, Fracture mechanics for piezoelectric ceramics, J. Mech. Phys. Solids 40, 739-765

[22] Tiersten, H. F., 1969, Linear piezoelectric plate vibrations, Plenum Press, New York

[23] Ting, T. C. T., 1988, Some identities and the structure of $\mathbf{N}_{i}$ in the Stroh formalism of anisotropic elasticity, Q. Appl. Math. 46, 109-120

[24] Ting, T. C. T., 1996, Anisotropic elasticity: Theory and applications, Oxford University Press, New York

[25] Wu, C. H., 1994, Regularly and singularly perturbed crack, Q. Appl. Math. 52, 529-543

[26] Zhang, T.-Y., Qian, C.-F., and Tong, P., 1998, Linear electro-elastic analysis of a cavity or a crack in a piezoelectric material, Int. J. Solids Structures 35 (17), 2121-2149 